\title{
ON THE WELL-POSEDNESS OF MATHEMATICAL MODELS FOR MULTICOMPONENT BIOFILMS
}

\author{
STEFANIE SONNER ${ }^{A}$, MESSOUD A. EFENDIEV $^{B}$ AND HERMANN J. EBERL ${ }^{C *}$
}

\begin{abstract}
Bacterial biofilms are microbial depositions on immersed surfaces. Their mathematical description leads to degenerate diffusion-reaction equations with two non-Fickian effects: (i) a porous medium equation like degeneracy where the biomass density vanishes, and (ii) a super-diffusion singularity if the biomass density reaches its threshold density. In the case of multi-species interactions, several such equations are coupled, both in the reaction terms and in the nonlinear diffusion operator. In this paper we generalize previous work on existence and uniqueness of solutions of this type of models and give a general, relatively easy to apply criterion for well-posedness. The use of the criterion is illustrated in several examples from the biofilm modeling literature.
\end{abstract}

\section{INTRODUCTION}

The dominant mode of microbial life in aquatic ecosystems are biofilm communities rather than planktonic cultures [1]. Biofilms are dense aggregations of microbial cells encased in a slimy extracellular matrix forming on biotic or abiotic surfaces (called substrata) in aqueous surroundings. Such multicellular communities are a very successful life form and able to tolerate harmful environmental impacts that would eradicate free floating individual cells [4, 21. Whenever environmental conditions allow for bacterial growth, microbial cells can attach to a substratum and switch to a sessile life form. They start to grow and divide and produce a gel-like layer of extracellular polymeric substances (EPS) often forming complex spatial structures. The self-produced EPS gives protection and allows survival in hostile environments. For instance, the mechanisms of antibiotic resistance in biofilm cultures are essentially different from those of free swimming cells, which makes it difficult to eradicate bacterial biofilm infections. The EPS retards diffusion of antibiotics and the antibiotic agents fail to penetrate into the inner core of the biofilm [4, 21, 5].

Biofilms play a significant role in various fields. They are beneficially used in environmental engineering technologies for groundwater protection and wastewater treatment. However, in most

2010 Mathematics Subject Classification. 35K65, 35K57, 92C17, 92D25.

Key words and phrases. biofilm; antibiotic disinfection; probiotic control; quorum-sensing; degenerate reactiondiffusion systems; well-posedness.

${ }^{A}$ BCAM - Basque Center for Applied Mathematics, Bilbao, Spain; University of Kaiserslautern, Felix-KleinCenter for Mathematics, Kaiserslautern, Germany

$B$ Institute of Computational Biology, Helmholtz Center Munich, Neuherberg, Germany

${ }^{C}$ Department of Mathematics and Statistics, University of Guelph, Guelph, Ontario, Canada

* corresponding author, E-mail: heberl@uoguelph.ca.

Until May 2014 the first author was supported by the ERC Advanced Grant FPT-246775 NUMERIWAVES. 
occurrences biofilm formations have negative effects. If they form on implants and natural surfaces in the human body they can provoke bacterial infections such as dental caries and otitis media [4]. Biofilm contamination can lead to health risks in food processing environments, and biofouling of industrial equipment or ships can cause severe economic defects for the industry [5, 26].

Mathematical models of biofilms have been studied for several decades. They range from traditional one-dimensional models that describe biofilms as homogeneous flat layers, to more recent two- and three-dimensional biofilm models that account for the spatial heterogeneity of biofilm communities. A variety of mathematical modeling concepts has been suggested, including discrete stochastic particle based models and deterministic continuum models, that are based on the description of the mechanical properties of biofilms [8, 26]. We are concerned with the latter, where biofilm and liquid surroundings are assumed to be continua, and its time evolution is governed by systems of deterministic PDEs. The first continuum model [27] was a one-dimensional biofilm growth model and essentially based on the assumption that biofilms are homogeneous flat layers. Such models serve well for engineering applications on the macro-scale (larger than $1 \mathrm{~cm}$ ) are, however, not capable to predict the often highly irregular spatial structure of microbial populations and the behavior of biofilms on the meso-scale (between $50 \mu \mathrm{m}$ and $1 \mathrm{~mm}$ ), the actual length scale of mature biofilms [8]. Biofilms can form mushroom-like caps and contain clusters and channels, where substrates can circulate. Cells in different regions of the biofilm live in diverse micro-environments and exhibit differing behavior 4 .

To capture the spatial heterogeneity of biofilms a higher dimensional biofilm growth model was proposed in [7], which is based on the interpretation of a biofilm as a continuous, spatially structured microbial population. The essential difficulty is to model the spatial spreading mechanism of biomass and to reproduce the growth characteristics of biofilms that have been observed in experiments [7]:

- Biofilm and aqueous surroundings are separated by a sharp interface.

- The biomass density is bounded by a known maximum value.

- Spatial spreading only takes place where the local biomass density approaches values close to its maximum possible value, while it does not occur in regions where the biomass density is low.

The mathematical model [7] is formulated as a system of highly non-linear reaction-diffusion equations for the biomass density and the concentration of a growth limiting nutrient, and is the prototype of the biofilm models we consider. While the substrate concentration satisfies a standard semi-linear reaction-diffusion equation the governing equation for the biomass density exhibits two non-linear diffusion effects. The biomass diffusion coefficient degenerates like the porous medium equation and shows super diffusion, which causes difficulties in the mathematical analysis of the model. It was shown by numerical experiments that the model is capable of predicting the heterogeneous spatial structure of biofilms and is in good agreement with experimental findings 
[7. In [11, 12] the model was studied analytically. In particular, the well-posedness of the model and the existence of a compact global attractor was shown.

The prototype single-species single-substrate model was extended to model biofilms which consist of several types of biomass and account for multiple dissolved substrates [25]. The model introduced in [5] describes the diffusive resistance of biofilms against the penetration by antibiotics. In [15] an amensalistic biofilm control system was modelled, where a beneficial biofilm controls the growth of a pathogenic biofilm. The structure of these multi-species models differs essentially from the mono-species model, and the analytical results for the prototype model could not all be carried over to the more involved multi-species case. In both articles, existence proofs for the solutions were given, and numerical studies presented, but the question of uniqueness of solutions remained unanswered in [5] and [15]. Recently, another multi-component biofilm model was proposed in [13], which describes quorum-sensing in growing biofilm communities. Quorum-sensing is a cellcell communication mechanism used by bacteria to coordinate behavior in groups. The model behavior was studied by numerical experiments in [13, 26], analytical questions were addressed in [26]. Compared to the multicomponent biofilm models [5, 15], the particularity of the quorumsensing model is, that adding the governing equations for the involved biomass components we recover exactly the mono-species biofilm growth model. Taking advantage of the known results for the prototype model the existence and uniqueness of solutions and the continuous dependence of solutions on initial data could be established in [26]. It is the first uniqueness result for multispecies reaction-diffusion models of biofilms that extend the single-species model [7]. We aim to show that the solution theory developed in [26] can be extended to other multicomponent biofilm models.

In Section 2, for the convenience of the reader, we introduce the prototype biofilm growth model and summarize important results on which our work is based. Multi-component extensions of the model are addressed in Section 3 As our main result, we formulate a general existence result for solutions of multicomponent biofilm models in Subsection 4.1, and the uniqueness of solutions is shown in Subsection 4.2. In Section 5 we verify that the solution theory applies to the multicomponent models of Section 3 . Finally, in Section 6 we present numerical simulations for the antibiotics model.

\section{Prototype Biofilm Growth Model}

The multi-dimensional biofilm growth model proposed in [7] is formulated as a non-linear reactiondiffusion system for the biomass density and the concentration of the growth controlling nutrient in a bounded domain $\Omega \subset \mathbb{R}^{n}, n=1,2,3$, where the boundary of the domain $\partial \Omega$ is piecewise smooth. In dimensionless form the substrate concentration $S$ is scaled with respect to the bulk concentration, and the biomass density is normalized with respect to the maximal bound for the cell density. Consequently, the dependent model variable $M$ represents the volume fraction occupied by biomass. The EPS is implicitly taken into account, in the sense that the biomass volume fraction $M$ describes the sum of biomass and EPS, assuming that their volume ratio is 
constant. Both unknown functions depend on the spatial variable $x \in \Omega$ and time $t \geq 0$, and satisfy the parabolic system

$$
\begin{aligned}
\partial_{t} S & =d_{S} \Delta S-k_{1} \frac{S M}{k_{2}+S} & & \Omega \times(0, \infty), \\
\partial_{t} M & =d \nabla \cdot(D(M) \nabla M)+k_{3} \frac{S M}{k_{2}+S}-k_{4} M & & \Omega \times(0, \infty), \\
\left.M\right|_{\partial \Omega} & =0,\left.\quad S\right|_{\partial \Omega}=1 & & \partial \times[0, \infty), \\
\left.M\right|_{t=0} & =M_{0},\left.\quad S\right|_{t=0}=S_{0} & & \Omega \times\{0\},
\end{aligned}
$$

where the constants $d, d_{S}$ and $k_{2}$ are positive, the constants $k_{1}, k_{3}$ and $k_{4}$ are non-negative. Furthermore, $\Delta$ denotes the Laplace operator, $\nabla$ the gradient operator with respect to the spatial variable $x$ and t the inner product in $\mathbb{R}^{n}$.

The solid region occupied by the biofilm as well as the liquid surroundings are assumed to be continua. The actual biofilm is described by the region $\Omega_{1}(t):=\{x \in \Omega \mid M(x, t)>0\}$, and the liquid area by $\Omega_{2}(t):=\{x \in \Omega \mid M(x, t)=0\}$, see Figure1. The substratum, on which the biofilm grows, is part of the boundary $\partial \Omega$.

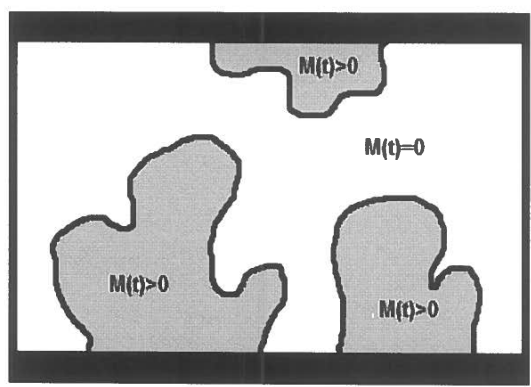

Figure 1. Biofilm Domain

The constants in System (1) have the following meaning, for further details and their typical values in applications we refer to [7].

\begin{tabular}{|c|l|}
\hline$d_{S}$ & substrate diffusion coefficient \\
$d$ & biomass motility constant \\
$k_{1}$ & maximum specific consumption rate \\
$k_{2}$ & Monod half saturation constant \\
$k_{3}$ & maximum specific growth rate \\
$k_{4}$ & biomass decay rate \\
$a, b$ & biomass spreading parameters \\
\hline
\end{tabular}

Biomass is produced due to the consumption of nutrients, which is described by Monod interaction functions. Natural cell death is also included in the model and given by the lysis rate $k_{4}$ in the equation for the biomass fraction. 
While the nutrient is dissolved in the domain and the substrate concentration satisfies a standard semi-linear reaction-diffusion equation, the spatial spreading of biomass is determined by the density-dependent diffusion coefficient

$$
D(M)=\frac{M^{a}}{(1-M)^{b}} \quad a, b \geq 1 .
$$

The biomass motility constant $d$ is small compared to the diffusion coefficient $d_{S}$ of the dissolved substrate, which reflects that the cells are to some extent immobilized in the EPS matrix. Accumulation of biomass leads to spatial expansion of the biofilm. We observe that the biomass diffusion coefficient vanishes when the total biomass approaches zero and blows up when the biomass density tends to its maximum value. The polynomial degeneracy $M^{a}$ is well-known from the porous medium equation and guarantees that spatial spreading is negligible for low values of $M$. Moreover, it yields the separation of biofilm and liquid phase, that is, a finite speed of interface propagation. Spreading of biomass takes place when and where the biomass fraction takes values close to its maximal value. When $M=1$ instantaneous spreading occurs, which is known as the effect of super diffusion. This singularity at $M=1$ of the biomass diffusion coefficient ensures the maximal bound for the biomass density.

It was shown in numerical experiments that the model (1) is in good agreement with experimental findings and is capable to reproduce the irregular, heterogeneous spatial structure of biofilms observed on the mesoscale [7, 12]. More precisely, the simulations show that the biofilm develops a rather regular, homogeneous structure if nutrients are nowhere limited in the system. On the other hand, when the nutrient supply is not symmetric and nutrients become limited the colonies grow in the direction of higher nutrient concentrations, which can lead to cluster and channel morphologies and mushroom-shaped architectures.

A solution theory for the prototype model (1) was developed in [12. In particular, the wellposedness was established and the existence of a compact global attractor was shown.

We recall the main results in [12]. The following theorem yields the existence and regularity results for the solutions (Theorem 3.1, [12]).

Theorem 1. We assume the initial data satisfies

$$
\begin{aligned}
& S_{0} \in L^{\infty}(\Omega) \cap H^{1}(\Omega),\left.\quad S_{0}\right|_{\partial \Omega}=1, \\
& M_{0} \in L^{\infty}(\Omega), \quad F\left(M_{0}\right) \in H_{0}^{1}(\Omega), \\
& 0 \leq S_{0} \leq 1, \quad 0 \leq M_{0} \text { in } \Omega, \quad\left\|M_{0}\right\|_{L^{\infty}(\Omega)}<1,
\end{aligned}
$$

where the function $F(v):=\int_{0}^{v} \frac{z^{a}}{(1-z)^{b}} d z$, for $0 \leq v<1$. Then, there exists a solution $(S, M)$ satisfying System (1) in the sense of distributions, and the solution belongs to the class

$$
\begin{aligned}
& M, S \in L^{\infty}(\Omega \times(0, \infty)) \cap C\left([0, \infty) ; L^{2}(\Omega)\right), \\
& F(M), S \in L^{\infty}\left((0, \infty) ; H^{1}(\Omega)\right) \cap C\left([0, \infty) ; L^{2}(\Omega)\right), \\
& 0 \leq S, M \leq 1 \text { in } \Omega \times(0, \infty), \quad\|M\|_{L^{\infty}(\Omega \times(0, \infty))}<1 .
\end{aligned}
$$


Moreover, it was shown that the solutions are $L^{1}(\Omega)$-Lipschitz continuous with respect to initial data, which implies its uniqueness. The following result recalls Theorem 3.2 in [12].

Proposition 1. Let $(S, M)$ and $(\widetilde{S}, \widetilde{M})$ be two solutions of System (1) corresponding to initial data $\left(S_{0}, M_{0}\right),\left(\widetilde{S}_{0}, \widetilde{M}_{0}\right)$ respectively, and the initial data satisfy the assumptions of the previous theorem. Then, the following estimate holds

$$
\|S(t)-\widetilde{S}(t)\|_{L^{1}(\Omega)}+\|M(t)-\widetilde{M}(t)\|_{L^{1}(\Omega)} \leq e^{c t}\left(\left\|S_{0}-\widetilde{S}_{0}\right\|_{L^{1}(\Omega)}+\left\|M_{0}-\widetilde{M}_{0}\right\|_{L^{1}(\Omega)}\right)
$$

for $t \geq 0$ and some constant $c \geq 0$.

\section{Multicomponent Biofilm Models}

The prototype biofilm growth model (1) was extended to incorporate further biofilm processes. It requires to distinguish different types of biomass and dissolved substrates and to include governing equations for these multiple biomass fractions and dissolved substrates in the model. In this section we discuss multi-component biofilm models, that were proposed and studied in [5, 6, 13, 15, 26] and recall the analytical results obtained for these models. The pattern of the multi-component biofilm models is essentially different from the prototype model, the equations for the biomass components are strongly coupled through the diffusion operators and the analytical results for the single-species model could not all be carried over. In [5] and [15] the behavior of solutions was studied in numerical simulations and the existence of solutions was established, but the question of uniqueness of solutions remained unanswered in both cases. The first uniqueness result for multispecies models was obtained in [26.

3.1. Antibiotic Disinfection of Biofilms. The first multi-species multi-substrate generalization of the prototype model (1) was suggested in [6]. In [5] existence results for the solutions were established and numerical simulations presented. The model describes a growing biofilm community and its disinfection by antimicrobial agents. Bacteria in biofilm populations are better protected than free floating cells and behave essentially different under antibiotic treatment. The EPS retards diffusion of antimicrobial agents into the biofilm, cells in the outer layers are attacked first while bacteria in the inner cores are well protected and continue to grow.

The dependent model variables are:

$$
\begin{array}{ll}
S & \text { nutrient concentration } \\
B & \text { concentration of the antimicrobial agent } \\
X & \text { volume fraction occupied by active biomass } \\
Y & \text { volume fraction occupied by inert biomass }
\end{array}
$$

The dissolved nutrient $S$ controls the growth of the biomass, and the antimicrobial agent $B$ regulates the disinfection process. As previously, the EPS is implicitly taken into account, and the total biomass fraction $M:=X+Y$ is normalized with respect to the maximum bound for the 
cell density. In dimensionless form the model is represented by the parabolic system

$$
\begin{aligned}
\partial_{t} S & =d_{S} \Delta S-k_{1} \frac{S X}{k_{2}+S} & & \Omega \times(0, \infty), \\
\partial_{t} B & =d_{B} \Delta B-\zeta_{1} B X & & \Omega \times(0, \infty), \\
\partial_{t} X & =d \nabla \cdot(D(M) \nabla X)+k_{3} \frac{S X}{k_{2}+S}-k_{4} X-\zeta_{2} B X & & \Omega \times(0, \infty), \\
\partial_{t} Y & =d \nabla \cdot(D(M) \nabla Y)+\zeta_{2} B X & & \Omega \times(0, \infty),
\end{aligned}
$$

with non-negative and bounded initial and boundary data

$$
\begin{array}{rrrrr}
\left.X\right|_{\partial \Omega}=0, & \left.Y\right|_{\partial \Omega}=0, & \left.S\right|_{\partial \Omega}=S_{r}, & \left.B\right|_{\partial \Omega}=B_{r} & \partial \Omega \times[0, \infty), \\
\left.X\right|_{t=0}=X_{0}, & \left.Y\right|_{t=0}=Y_{0}, & \left.S\right|_{t=0}=S_{0}, & \left.B\right|_{t=0}=B_{0} & \Omega \times\{0\},
\end{array}
$$

where we use the same notations as in (1). The additional constants $\zeta_{1}, \zeta_{2}$ and $d_{B}$ in (2) are positive and have the following meaning:

\begin{tabular}{|c|l|}
\hline$d_{B}$ & diffusion coefficient of antibiotics \\
$\zeta_{1}$ & antibiotics consumption rate \\
$\zeta_{2}$ & inert biomass production rate \\
\hline
\end{tabular}

Apart from the diffusion of the dissolved substrates and the death, growth and spatial spreading of biomass the disinfection mechanism is included in the model. During this process antibiotic agents are consumed and active biomass is directly converted into inert biomass, which is determined by the disinfection parameters $\zeta_{1}$ and $\zeta_{2}$. Like in the mono-species model the production of active biomass due to the consumption of nutrients is described by Monod reaction functions. In the absence of antimicrobial agents and inert biomass, the model reduces to the single species biofilm growth model (1).

In [5] numerical simulations were presented to illustrate the model behavior and to analyze the efficiency of different disinfection strategies. The numerical experiments show that cells in the outer layers of the biofilm are attacked first while cells in the inner scores remain protected and survive longer.

The following existence result for solutions of the antibiotics model (2) was shown in [5] (Theorem 2.3). Further analytical results were not obtained; in particular, the uniqueness of solutions remained unanswered.

Theorem 2. We assume the functions $B_{r}$ and $S_{r}$ are non-negative and belong to the class $L^{\infty}(\partial \Omega)$. Moreover, if the initial data $X_{0}, Y_{0}, S_{0}, B_{0}$ are non-negative, belong to $L^{\infty}(\Omega)$ and satisfy

$$
0 \leq S_{0} \leq 1 \quad \text { in } \Omega, \quad\left\|X_{0}+Y_{0}\right\|_{L^{\infty}(\Omega)}<1
$$


then, there exists a global solution of the antibiotics model; the functions $S, B, X$ and $Y$ belong to the space $L^{\infty}(\Omega \times(0, \infty))$, are non-negative and satisfy System (2) in distributional sense.

3.2. Amensalistic Biofilm Control System. The model of an amensalistic biofilm control system [15] extends the single-species probiotic model [16] and possesses a similar structure as the model of antibiotic disinfection. In [15] existence results for the solutions were established and numerical simulations presented.

The model describes how a beneficial biofilm controls the growth of a pathogenic biofilm community by alternating the environmental conditions. The probiotic biofilm modifies the local concentration of protonated lactic acids, which decreases the $\mathrm{pH}$ concentration and deteriorates the growth conditions for the pathogens, while the controlling bacteria are more tolerant to these changes. The dependent model variables are:

$$
\begin{array}{ll}
C & \text { concentration of protonated lactic acids } \\
P & \text { concentration of hydrogen ions } \\
X & \text { volume fraction occupied by pathogens } \\
Y & \text { volume fraction occupied by probiotics } \\
Z & \text { volume fraction occupied by inert biomass }
\end{array}
$$

In dimensionless form the model is represented by the parabolic system

$$
\begin{array}{ll}
\partial_{t} C=d_{C} \Delta C+\alpha_{1} X\left(\zeta_{1}-C\right)+\alpha_{2} Y\left(\zeta_{1}-C\right) & \Omega \times(0, \infty), \\
\partial_{t} P=d_{P} \Delta P+\alpha_{3} C\left(\zeta_{2}-P\right) & \Omega \times(0, \infty), \\
\partial_{t} X=d \nabla \cdot(D(M) \nabla X)+\mu_{1} \psi_{1}(C, P) X & \Omega \times(0, \infty), \\
\partial_{t} Y=d \nabla \cdot(D(M) \nabla Y)+\mu_{2} \psi_{2}(C, P) Y & \Omega \times(0, \infty), \\
\partial_{t} Z=d \nabla \cdot(D(M) \nabla Z)-\min \left\{0, \mu_{1} \psi_{1}(C, P) X\right\}-\min \left\{0, \mu_{2} \psi_{2}(C, P) Y\right\} & \Omega \times(0, \infty),
\end{array}
$$

with non-negative and bounded initial and boundary data

$$
\begin{aligned}
& \left.C\right|_{\partial \Omega}=C_{r},\left.\quad P\right|_{\partial \Omega}=P_{r},\left.\quad X\right|_{\partial \Omega}=0,\left.\quad Y\right|_{\partial \Omega}=0,\left.\quad Z\right|_{\partial \Omega}=0 \quad \partial \Omega \times[0, \infty), \\
& \left.C\right|_{t=0}=C_{0},\left.\quad P\right|_{t=0}=P_{0},\left.\quad X\right|_{t=0}=X_{0},\left.\quad Y\right|_{t=0}=Y_{0},\left.\quad Z\right|_{t=0}=Z_{0} \quad \Omega \times\{0\},
\end{aligned}
$$

where we use the same notations as in (1). The constants $d_{C}, d_{P}, \alpha_{1}, \alpha_{2}, \alpha_{3}, \mu_{i}$ and $\zeta_{i}, i=1,2$, are positive and have the following meaning: 


\begin{tabular}{|c|l|}
\hline$d_{C}$ & diffusion coefficient of protonated lactic acids \\
$d_{P}$ & diffusion coefficient of hydrogen ions \\
$\alpha_{1}$ & acid production rate by pathogens \\
$\alpha_{2}$ & acid production rate by probiotics \\
$\alpha_{3}$ & hydrogen ions production rate \\
$\mu_{1}$ & maximum growth rate of pathogens \\
$\mu_{2}$ & maximum growth rate of probiotics \\
$\zeta_{1}$ & acid saturation level \\
$\zeta_{2}$ & hydrogen ion saturation level \\
$\zeta_{i}^{1}$ & pathogen growth kinetics, $i=1, \ldots, 6$ \\
$\zeta_{i}^{2}$ & probiotics growth kinetics, $i=1, \ldots, 6$ \\
\hline
\end{tabular}

Inert probiotics and pathogens are not distinguished in the model. As previously, the EPS is implicitly taken into account, and the total biomass fraction $M:=X+Y+Z$ is normalized with respect to the maximum bound for the cell density. Protonated lactic acids $C$ are produced by both bacterial species until a saturation level is reached. The hydrogen ion concentration $P$ is related to the local $\mathrm{pH}$ value by $\mathrm{pH}=-\log P$. It increases, facilitated by the protonated lactic acids, until a threshold value is archived.

The growth and inhibition functions $\psi_{1}$ and $\psi_{2}$ are piecewise linear such that they are positive if $C$ and $P$ are small, and negative if $C$ or $P$ becomes large. Between the growth and inhibition range there is an extended neutral range. More precisely, the functions $\psi_{i}$ are piecewise linear and continuous. They are given for $\zeta_{5}^{i}>C \geq 0, \zeta_{6}^{i}>P \geq 0$ by

$$
\psi_{i}(C, P)=\min \left\{1-\frac{C}{h_{1}^{i}(C)}, 1-\frac{P}{h_{2}^{i}(P)}\right\}, \quad i=1,2,
$$

where $h_{1}^{i}$ and $h_{2}^{i}$ are defined as

$$
\begin{array}{ll}
h_{1}^{i}(C)=\zeta_{1}^{i} h\left(\zeta_{1}^{i}-C\right)+C h\left(C-\zeta_{1}^{i}\right) h\left(\zeta_{2}^{i}-C\right)+h\left(C-\zeta_{2}^{i}\right), & i=1,2, \\
h_{2}^{i}(P)=\zeta_{3}^{i} h\left(\zeta_{3}^{i}-P\right)+P h\left(P-\zeta_{3}^{i}\right) h\left(\zeta_{4}^{i}-P\right)+h\left(P-\zeta_{4}^{i}\right), \quad i=1,2 .
\end{array}
$$

Moreover, the function $h$ is given by

$$
h(s):=\left\{\begin{array}{ll}
1 & s>0, \\
\frac{1}{2} & s=0, \\
0 & s<0,
\end{array} \quad s \in \mathbb{R}\right.
$$

and the constants $\zeta_{j}^{1}$ and $\zeta_{j}^{2}, j=1, \ldots, 6$, are positive with $\zeta_{1}^{i}<\zeta_{2}^{i} \ll \zeta_{5}^{i}, \zeta_{3}^{i}<\zeta_{4}^{i} \ll \zeta_{6}^{i}, i=1,2$. For the probiotic strategy to be effective we require that $\zeta_{j}^{2}<\zeta_{j}^{1}, j=1, \ldots, 6[15]$.

Formally $\psi_{i}(C, P)$ can be extended to ranges $C>\zeta_{5}^{i}$ by $\psi_{1}(C, P)=\psi\left(\zeta_{5}^{i}, P\right)$ and similar for $P>\zeta_{6}^{i}, C<0, P<0$. Thus, the functions $\psi_{1,2}$ are bounded between two constants.

The mechanism of probiotic control is different from traditional antibiotic control strategies of biofilms, where the inner layers of the film are protected by the outer layers and the antibiotics 
fail to fully penetrate the biofilm. The numerical experiments for the probiotic biofilm model in 15. show that pathogens in the core of the biofilm, close to the substratum, are eradicated first. The structures of the antibiotics model (3) and the probiotics model (2) are similar and the existence of solutions of the probiotic model was shown by similar arguments (Theorem 3.3, [15]). For the proof of the following theorem we refer to [15]. As for the model of antibiotic disinfection, further analytical results were not obtained and the uniqueness of solutions remained unanswered.

Theorem 3. We assume the functions $C_{r}$ and $P_{r}$ belong to the class $L^{\infty}(\partial \Omega)$ and satisfy

$$
0 \leq C_{r} \leq \zeta_{1}, \quad 0 \leq P_{r} \leq \zeta_{2}
$$

Moreover, if the initial data $C_{0}, P_{0}, X_{0}, Y_{0}, Z_{0}$ are non-negative, belong to $L^{\infty}(\Omega)$ and satisfy

$$
0 \leq C_{0} \leq \zeta_{1}, \quad 0 \leq P_{0} \leq \zeta_{2} \quad \text { in } \Omega, \quad\left\|X_{0}+Y_{0}+Z_{0}\right\|_{L^{\infty}(\Omega)}<1
$$

then, there exists a global solution of the probiotics model, the functions $C, P, X, Y$ and $Z$ belong to $L^{\infty}(\Omega \times(0, \infty))$, are non-negative and satisfy system $\sqrt{3}$ in distributional sense.

3.3. Quorum-Sensing in Patchy Biofilm Communities. A model for quorum-sensing in growing biofilm communities was proposed and studied by numerical simulations in [13]. It extends the prototype biofilm growth model (1) and combines it with the model for quorum-sensing in planktonic cultures in [20]. Analytical aspects of the quorum-sensing model [13] were addressed in 26$]$.

Quorum-sensing is a cell-cell communication mechanism used by bacteria to coordinate gene expression and behavior in groups. Bacteria constantly produce low amounts of signaling molecules that are released into the environment. Accumulation of signaling molecules triggers a response by the cells and since the producing cells respond to their own signals the molecules are also called autoinducers [20, 14]. When the concentration of autoinducers locally passes a certain threshold, the cells are rapidly induced, and switch from a so-called down-regulated to an up-regulated state. In an up-regulated state they typically produce the signaling molecule at a highly increased rate 13 .

The dependent model variables are:

$S$ concentration of growth controlling substrate

$A$ concentration of autoinducers

$X$ volume fraction occupied by down-regulated cells

$Y$ volume fraction occupied by up-regulated cells 
In dimensionless form the model is represented by the parabolic system

$$
\begin{aligned}
\partial_{t} S & =d_{S} \Delta S-k_{1} \frac{S M}{k_{2}+S} & & \Omega \times(0, \infty), \\
\partial_{t} A & =d_{A} \Delta A-\gamma A+\alpha X+(\alpha+\beta) Y & & \Omega \times(0, \infty), \\
\partial_{t} X & =d \nabla \cdot(D(M) \nabla X)+k_{3} \frac{X S}{k_{2}+S}-k_{4} X-k_{5}|A|^{m} X+k_{5}|Y| & & \Omega \times(0, \infty), \\
\partial_{t} Y & =d \nabla \cdot(D(M) \nabla Y)+k_{3} \frac{Y S}{k_{2}+S}-k_{4} Y+k_{5}|A|^{m} X-k_{5}|Y| & & \Omega \times(0, \infty),
\end{aligned}
$$

with non-negative and bounded initial and boundary data

$$
\begin{array}{rlrrr}
\left.S\right|_{\partial \Omega}=1, & \left.A\right|_{\partial \Omega}=0, & \left.X\right|_{\partial \Omega}=1, & \left.Y\right|_{\partial \Omega}=0 & \partial \Omega \times[0, \infty), \\
\left.S\right|_{t=0}=S_{0}, & \left.A\right|_{t=0}=A_{0}, & \left.X\right|_{t=0}=X_{0}, & \left.Y\right|_{t=0}=Y_{0} & \Omega \times\{0\},
\end{array}
$$

where we use the same notations as in (1) and $|\cdot|$ denotes the absolute value. The constants $d_{A}$ and $\gamma$ are positive, $m \geq 1$, and $\alpha, \beta$ and $k_{5}$ are non-negative. Moreover, we require that $\alpha+\beta>\gamma$. Apart from the constants in the prototype model (1) the parameters in $(3)$ have the following meaning:

\begin{tabular}{|c|l|}
\hline$d_{A}$ & diffusion coefficient of autoinducers \\
$k_{5}$ & up-regulation rate \\
$\alpha$ & autoinducer production rate of down-regulated cells \\
$\beta$ & increased autoinducer production rate of up-regulated cells \\
$\gamma$ & abiotic decay rate of autoinducers \\
$m$ & polymerization exponent \\
\hline
\end{tabular}

The total biomass density $M=X+Y$ is normalized with respect to the maximum bound for the cell density and the EPS is implicitly taken into account. Assuming that induction switches the cells between down- and up-regulated states without changing their growth behavior we can assume that the spatial spreading of both biomass fractions is described by the same diffusion operator. The biomass motility constant $d$ is small compared to the diffusion coefficients $d_{S}$ and $d_{A}$ of the dissolved substrates. Like in the mono-species biofilm model, biomass is produced due to the consumption of nutrients, which is described by Monod reaction functions. Natural cell death is included and determined by the lysis rate $k_{4}$. If we do not distinguish between up-regulated and down-regulated cells in the model (4) we recover the prototype biofilm growth model (1) for the total biomass $M$ and the growth controlling nutrient $S$.

The autoinducer concentration $A$ is normalized with respect to the threshold concentration for induction. Down-regulated cells produce the signaling molecule at rate $\alpha$, while up-regulated cells produce it at the increased rate $\alpha+\beta$, where $\beta$ is one order of magnitude larger than $\alpha$. Due to an increase of the autoinducer concentration $A$ down-regulated cells are converted into up-regulated cells at rate $k_{5} A^{m}$. In applications typical values for the degree of polymerization are $2<m<3$ [13, 26. Up-regulated cells are converted back into down-regulated cells at constant rate $k_{5}$. If the molecule concentration $A<1$ the latter effect dominates, if $A>1$ up-regulation is super-linear. 
Moreover, abiotic decay of signaling molecules is taken into account in the model and determined by the constant rate $\gamma$.

The numerical simulations for the model in [26] indicate that quorum-sensing in spatially structured biofilm populations does not only depend on the local cell density of the population but also on mass transfer effects. Namely, the location of the cell colonies relative to each other and on the prescribed boundary conditions for the substrates.

The particularity of the quorum-sensing model is that adding the governing equations for the biomass components $X$ and $Y$ we recover exactly the mono-species biofilm growth model (1) for the total biomass $M$ and the nutrient concentration $S$. Using the known results for the prototype model a different approach than for the previous multi-component models was developed in [26], which led to a uniqueness result for the solutions. The following theorem states the well-posedness of the quorum-sensing model (4) (Theorem 3.5 and Theorem 3.11, [26]).

Theorem 4. Let the initial data satisfy $X_{0}, Y_{0}, A_{0} \in H_{0}^{1}(\Omega), S_{0} \in H^{1}(\Omega)$ such that $\left.S_{0}\right|_{\partial \Omega}=1$, and

$$
0 \leq S_{0}, X_{0}, Y_{0}, A_{0} \leq 1 \quad \text { in } \Omega, \quad\left\|X_{0}+Y_{0}\right\|_{L^{\infty}(\Omega)}<1
$$

Then, there exists a unique global solution of the quorum-sensing model (4),

$$
\begin{aligned}
& A, S, X, Y \in C\left([0, \infty) ; L^{2}(\Omega)\right) \cap L^{\infty}(\Omega \times[0, \infty)), \\
& A, S \in L^{2}\left((0, \infty) ; H^{1}(\Omega)\right) \\
& D(M) \nabla X, D(M) \nabla Y \in L^{2}\left((0, \infty) ; L^{2}\left(\Omega ; \mathbb{R}^{n}\right)\right)
\end{aligned}
$$

the functions $A, S, X$ and $Y$ are non-negative and satisfy system (4) in distributional sense.

\section{Well-Posedness of Multi-Component Models}

We consider multi-component biofilm models that are formulated as systems of reaction-diffusion equations for the concentrations of the dissolved substrates $S_{1}, \ldots, S_{k}$ and the biomass fractions $M_{1}, \ldots, M_{l}$ in a bounded domain $\Omega \subset \mathbb{R}^{n}, n=1,2,3$, with piecewise smooth boundary $\partial \Omega$. The models are of the form

$$
\begin{array}{rlrl}
\partial_{t} S_{i} & =d_{i} \Delta S_{i}+f_{i}(\underline{S}, \underline{M}) & \Omega \times(0, \infty), & i=1, \ldots, k \\
\partial_{t} M_{j}=d \nabla \cdot\left(D(M) \nabla M_{j}\right)+g_{j}(\underline{S}, \underline{M}) & \Omega \times(0, \infty), & j=1, \ldots, l, \\
\left.S_{i}\right|_{\partial \Omega}=\widehat{S}_{i} & \partial \Omega \times[0, \infty), & i=1, \ldots, k, \\
\left.M_{j}\right|_{\partial \Omega}=0 & \partial \Omega \times[0, \infty), & j=1, \ldots, l, \\
\left.S_{i}\right|_{t=0}=S_{i, 0} & \Omega \times\{0\}, & i=1, \ldots, k, \\
\left.M_{j}\right|_{t=0}=M_{j, 0} & \Omega \times\{0\}, & j=1, \ldots, l,
\end{array}
$$

where $(\underline{S}, \underline{M}):=\left(S_{1}, \ldots, S_{k}, M_{1}, \ldots, M_{l}\right)$ and $M:=\sum_{j=1}^{l} M_{j}$ denotes the total biomass. Moreover, the constants $d$ and $d_{1}, \ldots, d_{k}$ are positive, and the functions $\widehat{S}_{1}, \ldots \widehat{S}_{k}$ are non-negative and belong to the class $L^{\infty}(\partial \Omega)$. 
While the substrates $S_{1}, \ldots, S_{k}$ are dissolved in the domain and satisfy a standard semi-linear reaction-diffusion equation the spatial spreading of the biomass fractions is described by the density-dependent diffusion coefficient

$$
D(M):=\frac{M^{a}}{(1-M)^{b}}, \quad a, b \geq 1, M \in \mathbb{R} .
$$

The solid region as well as the liquid surroundings are assumed to be continua. The actual biofilm consists of several types of biomass and is described by the region $\Omega_{1}(t)=\{x \in \Omega \mid M(x, t)>0\}$. The liquid area is determined by $\Omega_{2}(t)=\{x \in \Omega \mid M(x, t)=0\}$.

If the functions $\widehat{S}_{i}$ are strictly positive, the boundary conditions imposed on the substrate concentrations in (5) reflect a constant unlimited substrate supply through the boundary of the considered domain. Similarly, keeping the substrate concentration $S_{i}$ equal to zero at the boundary, i.e., $\hat{S}_{i}=0$, enforces a removal of the substrate from the domain. The boundary conditions for the biomass fractions describe the situation that the growing biofilm is contained in the inner region of the domain, away from the boundary $\partial \Omega$. Such biofilms in the absence of a substratum are often called microbial flocs. Such bacterial aggregates enclosed in an EPS matrix are used in the industry for wastewater treatment and also occur in natural settings [22]. These are specific boundary conditions, primarily chosen for convenience. Boundary conditions of mixed type are often more appropriate in applications. Typically, Dirichlet conditions are specified on some part of the boundary, while Neumann or Robin conditions are imposed on the other parts. In particular, the substratum, on which the biofilm grows is impermeable for all dependent variables, which is described by homogeneous Neumann boundary values. The solution theory we develop in the following sections carries over to these more general boundary conditions as long as homogeneous Dirichlet boundary conditions are imposed for the biomass fractions on one part of the boundary. We assume the initial data $S_{1,0}, \ldots, S_{k, 0}, M_{1,0} \ldots, M_{l, 0}$ belong to the class $L^{\infty}(\Omega)$, are non-negative in $\Omega$ and satisfy

$$
\begin{array}{lll}
S_{i, 0} \in L^{\infty}(\Omega) \cap H^{1}(\Omega), & \left.S_{i, 0}\right|_{\partial \Omega}=\widehat{S}_{i}, & i=1, \ldots, k, \\
M_{j, 0}, F\left(M_{0}\right) \in H_{0}^{1}(\Omega), & \left\|M_{0}\right\|_{L^{\infty}(\Omega)}<1, & j=1, \ldots, l,
\end{array}
$$

where $M_{0}=\sum_{j=1}^{l} M_{j, 0}$ and

$$
F(M):=\int_{0}^{M} \frac{s^{a}}{(1-s)^{b}} d s \quad \text { for } 0 \leq M<1 .
$$

In the sequel, we denote by $Q_{T}:=\Omega \times[0, T), T>0$, the parabolic cylinder.

Definition 1. We call the vector-valued function $(\underline{S}, \underline{M})$ a solution of System (5) if its components belong to the class

$$
\begin{array}{lr}
S_{i}, M_{j} \in C\left([0, T] ; L^{2}(\Omega)\right) \cap L^{\infty}\left(Q_{T}\right), & i=1, \ldots, k, j=1, \ldots, l, \\
S_{i} \in L^{2}\left((0, T) ; H^{1}(\Omega)\right), & i=1, \ldots, k, \\
D(M) \nabla M_{j} \in L^{2}\left((0, T) ; L^{2}\left(\Omega ; \mathbb{R}^{n}\right)\right), & j=1, \ldots, l,
\end{array}
$$


for any $T>0$, and satisfy System (5) in distributional sense.

If $(\underline{S}, \underline{M})$ is a solution according to Definition 1 , then the equalities

$$
\begin{aligned}
\int_{\Omega} S_{i}(x, T) \varphi(x) d x & -\int_{\Omega} S_{i, 0}(x) \varphi(x) d x+d_{i} \int_{Q_{T}} \nabla S_{i}(x, t) \cdot \nabla \varphi(x) d t d x \\
& =\int_{Q_{T}} f_{i}(\underline{S}(x, t), \underline{M}(x, t)) \varphi(x) d t d x, \quad i=1, \ldots, k, \\
\int_{\Omega} M_{j}(x, T) \varphi(x) d x & -\int_{\Omega} M_{j, 0}(x) \varphi(x) d x+d \int_{Q_{T}} D(M(x, t)) \nabla M_{j}(x, t) \cdot \nabla \varphi(x) d t d x \\
& =\int_{Q_{T}} g_{j}(\underline{S}(x, t), \underline{M}(x, t)) \varphi(x) d t d x, \quad j=1, \ldots, l,
\end{aligned}
$$

hold for all test-functions $\varphi \in C_{0}^{\infty}(\Omega)$ and almost every $T>0$.

4.1. Existence. To prove the existence of solutions of the degenerate problem (5) we consider smooth regular approximations and show that the solutions of the approximating systems converge to the solution of the degenerate problem when the regularization parameter tends to zero. The ideas are based on the method developed in [12] for the mono-species model and the ideas applied in [5], 15] and 26] for multi-species biofilm models.

For small $\epsilon>0$ we define the non-degenerate approximations for Problem (5) by

$$
\begin{array}{rrr}
\partial_{t} S_{i}=d_{i} \Delta S_{i}+f_{i}(\underline{S}, \underline{M}) & \Omega \times(0, \infty), & i=1, \ldots, k, \\
\partial_{t} M_{j}=d \nabla \cdot\left(D_{\epsilon}(M) \nabla M_{j}\right)+g_{j}(\underline{S}, \underline{M}) & \Omega \times(0, \infty), & j=1, \ldots, l, \\
\left.S_{i}\right|_{\partial \Omega}=\widehat{S}_{i} & \partial \Omega \times[0, \infty), & i=1, \ldots, k, \\
\left.M_{j}\right|_{\partial \Omega}=0 & \partial \Omega \times[0, \infty), & j=1, \ldots, l, \\
\left.S_{i}\right|_{t=0}=S_{i, 0} & \Omega \times\{0\}, & i=1, \ldots, k, \\
\left.M_{j}\right|_{t=0}=M_{j, 0} & \Omega \times\{0\}, & j=1, \ldots, l,
\end{array}
$$

where the regularized diffusion coefficient $D_{\epsilon}$ is defined by

$$
D_{\epsilon}(M):=\left\{\begin{array}{cl}
\epsilon^{a} & M<0, \\
\frac{(M+\epsilon)^{a}}{(1-M)^{b}} & 0 \leq M \leq 1-\epsilon, \quad M \in \mathbb{R} . \\
\frac{1}{\epsilon^{b}} & M \geq 1-\epsilon,
\end{array}\right.
$$

Adding the equations for the biomass fractions leads to the system

$$
\begin{array}{ll}
\partial_{t} S_{i}=d_{i} \Delta S_{i}+f_{i}(\underline{S}, \underline{M}) & \Omega \times(0, \infty), \quad i=1, \ldots, k, \\
\partial_{t} M=d \nabla \cdot\left(D_{\epsilon}(M) \nabla M\right)+\sum_{j=1}^{l} g_{j}(\underline{S}, \underline{M}) & \Omega \times(0, \infty),
\end{array}
$$

for the total biomass $M$ and the dissolved substrates $S_{1}, \ldots, S_{k}$.

We denote by $\left(\underline{M}^{\epsilon}, \underline{S}^{\epsilon}\right)=\left(S_{1}^{\epsilon}, \ldots, S_{k}^{\epsilon}, M_{1}^{\epsilon}, \ldots, M_{l}^{\epsilon}\right)$ the solutions of the auxiliary systems (7). The well-posedness of the model (5) will be shown under the following hypotheses, which will be justified for each of the multicomponent models in Subsection 5 . 
$\left(\mathrm{A}_{1}\right)$ There exists $\epsilon_{0}^{\prime}>0$ such that all components of the solutions $\left(\underline{M}^{\epsilon}, \underline{S}^{\epsilon}\right)$ of the nondegenerate approximations are non-negative and uniformly bounded for all $\epsilon<\epsilon_{0}^{\prime}$.

$\left(\mathrm{A}_{2}\right)$ There exists a unique solution for the total biomass $M$ and the functions $f_{1}, \ldots, f_{k}$ and $g_{1}, \ldots, g_{l}$, satisfy

$$
\begin{array}{ll}
f_{i}(x)-f_{i}(y) \leq C\left(\sum_{m=1}^{k+l} x_{m}-y_{m}\right) & i=1, \ldots, k, \\
g_{j}(x)-g_{j}(y) \leq C\left(\sum_{m=1}^{k+l} x_{m}-y_{m}\right) & j=1, \ldots, l,
\end{array}
$$

for some constant $C \geq 0$ and $x, y$ in bounded subsets of

$$
\mathbb{R}_{+}^{k+l}=\left\{x \in \mathbb{R}^{k+l}: x_{i} \geq 0, i=1, \ldots, k+l\right\} .
$$

Remark 1. If hypothesis $\left(A_{1}\right)$ is satisfied a sufficient condition for the uniqueness of the total biomass $M$ is the following:

$$
\sum_{i=1}^{k}\left|f_{i}(\underline{S}, \underline{M})-f_{i}(\underline{\widetilde{S}}, \underline{\widetilde{M}})\right|+\left|\sum_{j=1}^{l} g_{j}(\underline{S}, \underline{M})-g_{j}(\underline{\widetilde{S}}, \underline{\widetilde{M}})\right| \leq c\left(|M-\widetilde{M}|+\sum_{i=1}^{k}\left|S_{i}-\widetilde{\widetilde{S}}_{i}\right|\right),
$$

for $(\underline{S}, \underline{M})$ and $(\underline{\widetilde{S}}, \underline{\widetilde{M}})$ in bounded subsets of $\mathbb{R}_{+}^{k+l}$ and some constant $c \geq 0$, where $M:=$ $\sum_{j=1}^{l} M_{j}, \widetilde{M}:=\sum_{j=1}^{l} \widetilde{M}_{j}$. In this case, the uniqueness of system (8) can be shown as for the prototype model in [12] (see Lemma 3.4, [12]).

First, we show that the total biomass $M^{\epsilon}$ is uniformly bounded away from the singularity for all sufficiently small $\epsilon>0$.

Lemma 1. Let the assumption $\left(\mathrm{A}_{1}\right)$ be satisfied and the initial data belong to the class (6). Then, there exists $0<\eta<1$ and $\epsilon_{0}>0$ such that the constant $1-\eta$ is an upper solution for the total biomass $M^{\epsilon}$ for all $\epsilon<\epsilon_{0}$.

Proof. Let $\epsilon_{0}^{\prime}>0$ be as in $\left(\mathrm{A}_{1}\right)$. To show the lemma we construct a suitable barrier function and consider the elliptic problem

$$
\begin{array}{cr}
\Delta \varphi(x)=-c_{1}, & x \in \Omega, \\
\left.\varphi(x)\right|_{\partial \Omega}=c_{2}, & x \in \partial \Omega,
\end{array}
$$

where the constants $c_{1}$ and $c_{2}$ are defined by

$$
\begin{aligned}
& c_{1}:=\sup _{0<\epsilon<\epsilon_{0}^{\prime}}\left\{\left\|\sum_{j=1}^{l} f_{j}\left(\underline{S}^{\epsilon}, \underline{M}^{\epsilon}\right)\right\|_{L^{\infty}\left(Q_{T}\right)}\right\}, \\
& c_{2}:=\sup _{0<\epsilon<\epsilon_{0}^{\prime}}\left\{\left\|F^{\epsilon}\left(M_{0}\right)\right\|_{L^{\infty}(\Omega)}\right\},
\end{aligned}
$$

and $F_{\epsilon}\left(M_{0}\right):=\int_{0}^{M_{0}} \frac{(s+\epsilon)^{a}}{(1-s)^{b}} d s$, if $M_{0} \leq 1-\epsilon$. Defining the constant $c_{1}$ we use the hypotheses $\left(A_{1}\right)$ and $\left(A_{2}\right)$ and remark that there exists $0<\epsilon_{1} \leq \epsilon_{0}^{\prime}$ such that the constant $c_{2}$ can be chosen uniform 
for all $\epsilon<\epsilon_{1}$. Moreover, the solution $\varphi$ is bounded on $\Omega$, and by the maximum principle follows $\varphi \geq c_{2}$ in $\Omega$.

For $\epsilon<\epsilon_{1}$ we define $Z_{\epsilon}:=F_{\epsilon}^{-1}(\varphi)$ and observe that

$$
\partial_{t} Z_{\epsilon}-\Delta\left(F_{\epsilon}\left(Z_{\epsilon}\right)\right)=c_{1}=\sup _{0<\epsilon<\epsilon_{1}}\left\{\left\|\sum_{j=1}^{l} f_{j}\left(\underline{S}^{\epsilon}, \underline{M}^{\epsilon}\right)\right\|_{L^{\infty}\left(Q_{T}\right)}\right\} \geq \partial_{t} M^{\epsilon}-\Delta\left(F_{\epsilon}\left(M^{\epsilon}\right)\right)
$$

in $Q_{T}$. Moreover, the boundary conditions imply

$$
\left.Z_{\epsilon}\right|_{\partial \Omega}=\left.F_{\epsilon}^{-1}(\varphi)\right|_{\partial \Omega}=F_{\epsilon}^{-1}\left(c_{2}\right) \geq\left. M^{\epsilon}\right|_{\partial \Omega}=0
$$

and the initial data satisfies

$$
\left.Z_{\epsilon}\right|_{t=0}=\left.F_{\epsilon}^{-1}(\varphi)\right|_{t=0} \geq F_{\epsilon}^{-1}\left(c_{2}\right) \geq F_{\epsilon}^{-1}\left(F_{\epsilon}\left(M_{0}\right)\right)=M_{0}
$$

where we used the monotonicity of the function $F_{\epsilon}^{-1}$. Consequently, the function $Z_{\epsilon}$ is an upper solution for the total biomass $M^{\epsilon}$. Using the fact that $\varphi$ is bounded in $\Omega$ and that $F_{\epsilon}$ converges pointwise to infinity in the interval $(0,1)$, we conclude that there exist $0<\epsilon_{0} \leq \epsilon_{1}$ and $\eta \in(0,1)$ such that $M^{\epsilon} \leq Z_{\epsilon}=F_{\epsilon}^{-1}(\varphi)<1-\eta$ for all $\epsilon<\epsilon_{0}$.

We particularly emphasize that the non-degenerate approximations for the total biomass $M^{\epsilon}$ are uniformly bounded away from the singularity,

$$
\left\|M^{\epsilon}(\cdot, t)\right\|_{L^{\infty}(\Omega)} \leq 1-\eta \quad \forall \epsilon<\epsilon_{0}, t \geq 0
$$

if the initial data satisfies $\left\|M_{0}\right\|_{L^{\infty}(\Omega)}<1$.

Lemma 2. We assume the assumption $\left(\mathrm{A}_{1}\right)$ is satisfied and the initial data belong to the class (6). Then, for all sufficiently small $\epsilon>0$ there exists a unique solution $\left(\underline{S}^{\epsilon}, \underline{M}^{\epsilon}\right)$ of the auxiliary system (7) satisfying

$$
\begin{aligned}
& S_{i}^{\epsilon}, M_{j}^{\epsilon} \in L^{2}\left((0, T) ; H^{1}(\Omega)\right) \cap C\left([0, T] ; L^{2}(\Omega)\right) \cap L^{\infty}\left(Q_{T}\right), \\
& \partial_{t} S_{i}^{\epsilon}, \partial_{t} M_{j}^{\epsilon} \in L^{2}\left((0, T) ; H^{-1}(\Omega)\right), \quad i=1, \ldots, k, j=1, \ldots, l .
\end{aligned}
$$

Moreover, the solutions are uniformly bounded with respect to the regularization parameter $\epsilon>0$, and satisfy the estimates

$$
\begin{aligned}
& \max _{t \in[0, T]}\left\|S_{i}^{\epsilon}(\cdot, t)\right\|_{L^{2}(\Omega)}+\left\|S_{i}^{\epsilon}\right\|_{L^{2}\left((0, T) ; H^{1}(\Omega)\right)}+\left\|\partial_{t} S_{i}^{\epsilon}\right\|_{L^{2}\left((0, T) ; H^{-1}(\Omega)\right)} \leq C\left(1+\left\|S_{i, 0}\right\|_{L^{2}(\Omega)}\right), \\
& \max _{t \in[0, T]}\left\|M_{j}^{\epsilon}(\cdot, t)\right\|_{L^{2}(\Omega)}+\left\|M_{j}^{\epsilon}\right\|_{L^{2}\left((0, T) ; H_{0}^{1}(\Omega)\right)}+\left\|\partial_{t} M_{j}^{\epsilon}\right\|_{L^{2}\left((0, T) ; H^{-1}(\Omega)\right)} \leq C_{\epsilon}\left(1+\left\|M_{j, 0}\right\|_{L^{2}(\Omega)}\right),
\end{aligned}
$$

$i=1, \ldots, k, j=1, \ldots, l$, for $t>0$ and some constants $C_{\epsilon}, C \geq 0$, where the constant $C$ is independent of $\epsilon>0$. The solutions are Hölder-continuous

$$
S_{i}^{\epsilon} \in C^{\alpha, \frac{\alpha}{2}}\left(Q_{T}\right), M_{j}^{\epsilon} \in C^{\alpha_{\epsilon}, \frac{\alpha_{\epsilon}}{2}}\left(Q_{T}\right), \quad i=1, \ldots, k, j=1, \ldots, l,
$$

where constants $\alpha_{\epsilon}$ and $\alpha$ are positive. The Hölder exponent $\alpha_{\epsilon}$ depends on the parameter $\epsilon$, the data and uniform bound of the approximate solutions only, the constant $\alpha$ is independent of $\epsilon>0$. 
Proof. If the initial data $\left(\underline{M}_{0}, \underline{S}_{0}\right)$ belongs to the class (6), Lemma 1 implies that there exists $\epsilon_{0}>0$ and $\eta \in(0,1)$ such that the approximate solutions for the total biomass density $M^{\epsilon}$ satisfy $M^{\epsilon}<1-\eta$ in $Q_{T}$ for all $\epsilon<\epsilon_{0}$, where the constant $\eta$ is independent of $\epsilon$. This implies that the diffusion coefficient $D_{\epsilon}\left(M^{\epsilon}\right)$ is positive and uniformly bounded from above by a constant, which is independent of $\epsilon$. Indeed, for all $\epsilon<\min \left\{\eta, \epsilon_{0}\right\}$ we obtain

$$
\epsilon^{a} \leq D_{\epsilon}\left(M^{\epsilon}(x, t)\right)=\frac{\left(M^{\epsilon}(x, t)+\epsilon\right)^{a}}{\left(1-M^{\epsilon}(x, t)\right)^{b}} \leq \frac{(1-\eta+\epsilon)^{a}}{(1-(1-\eta))^{b}} \leq \frac{1}{\eta^{b}}, \quad(x, t) \in Q_{T},
$$

which shows that $D_{\epsilon}\left(M^{\epsilon}\right) \in L^{\infty}\left(Q_{T}\right)$ and $D_{\epsilon}\left(M^{\epsilon}\right)$ is strictly positive. Hence, for all sufficiently small $\epsilon>0$ the semi-linear auxiliary system $(7)$ is regular and uniformly parabolic.

The functions $S_{i}^{\epsilon}$ and $M_{j}^{\epsilon}$ are uniformly bounded with respect to the regularization parameter $\epsilon>0$ by Hypothesis $\left(\mathrm{A}_{1}\right)$, which implies that the interaction functions $f_{i}\left(\underline{S}^{\epsilon}, \underline{M}^{\epsilon}\right)$ and $g_{j}\left(\underline{S}^{\epsilon}, \underline{M}^{\epsilon}\right)$ are uniformly bounded in $Q_{T}, i=1, \ldots, k, j=1, \ldots, l$. By standard arguments follow the existence and uniqueness of the approximate solutions $\left(\underline{S}^{\epsilon}, \underline{M}^{\epsilon}\right)$, the solutions belong to the class stated in the lemma and satisfy the given estimates (see Section 11.1 in [23]). Moreover, the Hölder-continuity of solutions follows from Theorem 10.1, Chapter III in [17].

Due to the uniform boundedness of the approximate solutions the components $S_{1}^{\epsilon}, \ldots, S_{k}^{\epsilon}$, satisfy the parabolic equation

$$
\partial_{t} S_{i}^{\epsilon}-d_{i} \Delta S_{i}^{\epsilon}=H_{i}^{\epsilon}, \quad i=1, \ldots, k,
$$

where the function $H_{i}^{\epsilon}$ is uniformly bounded, $\left\|H_{i}^{\epsilon}\right\|_{L^{\infty}\left(Q_{T}\right)} \leq c_{i}$ for some constant $c_{i} \geq 0, i=$ $1, \ldots, k$. Hence, the constants in the estimates for the components $S_{1}^{\epsilon}, \ldots, S_{k}^{\epsilon}$ can be chosen uniform with respect to the regularization parameter $\epsilon>0$.

We will show that the limit of the solutions of the non-degenerate approximations $\left(\underline{S}^{\epsilon}, \underline{M}^{\epsilon}\right)$ yields a solution of the degenerate problem $(\underline{S}, \underline{M})$. To show the convergence we treat the region, where the total biomass density is small, and its complement in $Q_{T}$ separately. For $\delta \in(0,1)$ and sufficiently small $\epsilon_{0}>0$ we define the domains

$$
Q_{\delta, T}:=\left\{(x, t) \in Q_{T} \mid M^{\epsilon}(t, x)<\delta \forall \epsilon<\epsilon_{0}\right\}
$$

and $Q_{\delta, T}^{c}:=Q_{T} \backslash \overline{Q_{\delta, T}}$. We note that both sets are open due to the Hölder-continuity of the solutions.

Remark 2. Restricted to the domain $Q_{\delta, T}^{c}$ the solutions $M_{1}^{\epsilon}, \ldots, M_{l}^{\epsilon}$ satisfy the estimates in Lemma 2 uniformly. To be more precise, the constant $C_{\epsilon}$ in the inequality and the Hölder exponent $\alpha_{\epsilon}$ are independent of $\epsilon>0$ for the family of approximate solutions $\widetilde{M}_{j}^{\epsilon}$, where $\widetilde{M}_{j}^{\epsilon}:=\left.M_{j}^{\epsilon}\right|_{Q_{\delta, T}^{c}}$, $j=1 \ldots, l$.

Indeed, if $\epsilon>0$ is sufficiently small, then $M^{\epsilon}>\delta$ in the region $Q_{\delta, T}^{c}$. Consequently, the diffusion coefficient restricted to the domain $Q_{\delta, T}^{c}$ is uniformly bounded from above and below by a positive constant which is independent of $\epsilon>0$,

$$
\left(\frac{\delta}{2}\right)^{a} \leq\left(\frac{\delta}{2}+\epsilon\right)^{a} \leq D_{\epsilon}\left(M^{\epsilon}(x, t)\right)=\frac{\left(M_{\epsilon}(x, t)+\epsilon\right)^{a}}{\left(1-M_{\epsilon}(x, t)\right)^{b}} \leq \frac{1}{\eta^{b}}, \quad(x, t) \in Q_{\delta, T}^{c} .
$$


Solutions of non-degenerate parabolic equations of second order with coefficients in $L^{\infty}(\Omega)$ satisfy the estimates stated in the lemma, and the bounds are determined in terms of the coefficients of the equation (see [17], Chapter V). Consequently, the estimates in the region $Q_{\delta, T}^{c}$ are uniform and do not dependent on $\epsilon>0$.

We will use Lemma 2 to pass to the limit in the region $Q_{\delta, T}^{c}$. To pass to the limit in the region $Q_{\delta, T}$ requires further uniform estimates for the family of approximate solutions.

Lemma 3. Let Hypothesis $\left(\mathrm{A}_{1}\right)$ be satisfied and the initial data belong to the class $(6)$. If $\epsilon_{0}>0$ is sufficiently small, the product $\sqrt{D_{\epsilon}\left(M^{\epsilon}\right)} \nabla M_{j}^{\epsilon}$ is uniformly bounded in $L^{2}\left(Q_{T} ; \mathbb{R}^{n}\right)$ for all $\epsilon<\epsilon_{0}$. Moreover, the approximate solutions satisfy $M_{j}^{\epsilon}(\cdot, t) \in H^{s}(\Omega)$ for some $s>0$ and almost every $t \in[0, T]$, and

$$
\left\|M_{j}^{\epsilon}\right\|_{L^{2}\left((0, T) ; H^{s}(\Omega)\right)} \leq C \quad \forall 0<\epsilon<\epsilon_{0}, j=1, \ldots, l,
$$

where the constant $C \geq 0$ is independent of the regularization parameter $\epsilon$.

Proof. Multiplying the governing equation for $M_{j}^{\epsilon}$ of System 77 by $M_{j}^{\epsilon}$ and integrating over $\Omega$ we obtain

$$
\begin{aligned}
\frac{1}{2} \frac{d}{d t}\left\|M_{j}^{\epsilon}(\cdot, t)\right\|_{L^{2}(\Omega)}^{2} & +d\left\langle D_{\epsilon}\left(M^{\epsilon}(\cdot, t)\right) \nabla M_{j}^{\epsilon}(\cdot, t), \nabla M_{j}^{\epsilon}(\cdot, t)\right\rangle_{L^{2}\left(\Omega ; \mathbb{R}^{n}\right)} \\
& =\int_{\Omega} M_{j}^{\epsilon}(x, t) g_{j}\left(\underline{S}^{\epsilon}(x, t), \underline{M}^{\epsilon}(x, t)\right) d x \leq C,
\end{aligned}
$$

for some constant $C \geq 0$. Due to Lemma 1 the constant $C$ is independent of $\epsilon>0$. If we integrate this inequality from 0 to $T$ follows the first statement of the lemma.

Furthermore, for sufficiently small $\epsilon>0$ we observe that $M_{j}^{\epsilon} \leq M^{\epsilon} \leq 1-\eta$ in $Q_{T}$ and consequently,

$$
\left(M_{j}^{\epsilon}(x, t)\right)^{a} \leq D_{\epsilon}\left(M_{j}^{\epsilon}(x, t)\right)=\frac{\left(M_{j}^{\epsilon}(x, t)+\epsilon\right)^{a}}{\left(1-M_{j}^{\epsilon}(x, t)\right)^{b}} \leq \frac{\left(M^{\epsilon}(x, t)+\epsilon\right)^{a}}{\left(1-\left(M^{\epsilon}(x, t)\right)\right)^{b}}=D_{\epsilon}\left(M^{\epsilon}(x, t)\right),
$$

for $(x, t) \in Q_{T}, j=1, \ldots, l$. This implies the estimate

$$
\int_{\Omega}\left(M_{j}^{\epsilon}(x, t)\right)^{a}\left\|\nabla M_{j}^{\epsilon}(x, t)\right\|^{2} d x \leq \int_{\Omega} D_{\epsilon}\left(M^{\epsilon}(x, t)\right)\left\|\nabla M_{j}^{\epsilon}(x, t)\right\|^{2} d x \leq C,
$$

for some constant $C \geq 0$, which is independent of the regularization parameter $\epsilon>0$. This shows that $\left(M_{j}^{\epsilon}\right)^{\frac{a}{2}}(\cdot, t) \nabla M_{j}^{\epsilon}(\cdot, t) \in L^{2}\left(\Omega ; \mathbb{R}^{n}\right)$ or equivalently, $\left(M_{j}^{\epsilon}\right)^{\frac{a}{2}+1}(t) \in H^{1}(\Omega)$ for almost every $t \in(0, T]$. Finally, if a function satisfies $\varphi^{\beta} \in H^{1}(\Omega)$ for some $\beta>1$, then $\varphi \in W^{s, 2 \beta}(\Omega)$ holds for all $s \leq \frac{1}{\beta}$ (see [10, Lemma 1.3). This implies that $M_{j}^{\epsilon}(\cdot, t) \in W^{s, 2\left(\frac{a}{2}+1\right)}(\Omega)$ for $s \leq \frac{1}{\frac{a}{2}+1}$. Since the domain $\Omega$ is bounded and $a \geq 1$ the embedding $W^{s, 2+a}(\Omega) \hookrightarrow H^{s}(\Omega)$ is continuous and we obtain $M_{j}^{\epsilon}(\cdot, t) \in H^{s}(\Omega)$ for some positive $s>0$. In particular, the family of approximate solutions $M_{j}^{\epsilon}, j=1, \ldots, l$, is uniformly bounded in the Hilbert space $L^{2}\left((0, T) ; H^{s}(\Omega)\right)$.

Lemma 4. We assume Hypothesis $\left(\mathrm{A}_{1}\right)$ is satisfied and the initial data belong to the class (6). Then, there exist functions

$$
\begin{array}{ll}
S_{i}^{*} \in L^{\infty}\left(Q_{T}\right) \cap L^{2}\left((0, T) ; H_{0}^{1}(\Omega)\right), & i=1, \ldots, k, \\
M_{j}^{*} \in L^{\infty}\left(Q_{T}\right) \cap L^{2}\left((0, T) ; H^{s}(\Omega)\right), & j=1, \ldots, l,
\end{array}
$$


and a sequence $\left(\epsilon_{k}\right)_{k \in \mathbb{N}}$ tending to zero as $k \rightarrow \infty$, such that the solutions of the auxiliary systems (7) converge weakly

$$
S_{i}^{\epsilon_{k}} \rightarrow S_{i}^{*} \text { in } L^{2}\left((0, T) ; H_{0}^{1}(\Omega)\right), \quad M_{j}^{\epsilon_{k}} \rightarrow M_{j}^{*} \text { in } L^{2}\left((0, T) ; H^{s}(\Omega)\right),
$$

and strongly

$$
M_{j}^{\epsilon_{k}} \rightarrow M_{j}^{*}, \quad S_{i}^{\epsilon_{k}} \rightarrow S_{i}^{*} \quad \text { in } C\left([0, T] ; L^{2}(\Omega)\right)
$$

when $k$ tends to infinity.

Proof. We prove the convergence and existence of the limit for the biomass fractions $M_{1}^{*}, \ldots, M_{l}^{*}$, the arguments are similar for the substrate concentrations $S_{1}^{*}, \ldots, S_{k}^{*}$. For sufficiently small $\epsilon>0$ the family of approximate solutions $M_{j}^{\epsilon}$ is uniformly bounded in the Hilbert space $L^{2}\left((0, T) ; H^{s}(\Omega)\right)$ for some $s>0$ by Lemma 3 . Consequently, there exists an element $M_{j}^{*} \in L^{2}\left((0, T) ; H^{s}(\Omega)\right)$ and a sequence $\left(\epsilon_{k}\right)_{k \in \mathbb{N}}$ tending to zero as $k \rightarrow \infty$ such that the sequence $\left(M_{j}^{\epsilon_{k}}\right)_{k \in \mathbb{N}}$ converges weakly to $M_{j}^{*}$ in $L^{2}\left((0, T) ; H^{s}(\Omega)\right), j=1, \ldots, l$.

Furthermore, Lemma3 implies that the product $\sqrt{D_{\epsilon}\left(M^{\epsilon}\right)} \nabla M_{j}^{\epsilon}$ is uniformly bounded in $L^{2}\left(Q_{T} ; \mathbb{R}^{n}\right)$, and that the diffusion coefficient satisfies $D_{\epsilon}\left(M^{\epsilon}\right) \in L^{\infty}\left(Q_{T}\right)$. Consequently, we obtain

$$
\left\|D_{\epsilon}\left(M^{\epsilon}\right) \nabla M_{j}^{\epsilon}\right\|_{L^{2}\left(Q_{T} ; \mathbb{R}^{n}\right)}^{2} \leq\left\|D_{\epsilon}\left(M^{\epsilon}\right)\right\|_{L^{\infty}\left(Q_{T}\right)}\left\|\sqrt{D_{\epsilon}\left(M^{\epsilon}\right)} \nabla M_{j}^{\epsilon}\right\|_{L^{2}\left(Q_{T} ; \mathbb{R}^{n}\right)}^{2} \leq c,
$$

for some constant $c \geq 0$, which is independent of $\epsilon>0$. This proves the uniform boundedness of the derivatives $\partial_{t} M_{j}^{\epsilon}$ in $L^{2}\left((0, T) ; H^{-1}(\Omega)\right), j=1, \ldots, l$.

By Theorem 1.5, Chapter II in [3] now follows the strong convergence of the sequence of approximate solutions in the space $C\left([0, T] ; L^{2}(\Omega)\right)$.

It remains to show that the limits of the approximate solutions yield a solution of the degenerate problem.

Theorem 5. If Hypothesis $\left(\mathrm{A}_{1}\right)$ is satisfied and the initial data belong to the class (6), the limits $S_{1}^{*}, \ldots, S_{k}^{*}$, and $M_{1}^{*}, \ldots, M_{l}^{*}$, of the solutions of the non-degenerate approximations in Lemma 4 are weak solutions of the degenerate system (5).

Proof. We show that we can pass to the limit $\epsilon \rightarrow 0$ in the distributional formulation of the nondegenerate auxiliary system (7). Since the arguments are the same or simplify for the substrate concentrations $S_{1}^{*}, \ldots, S_{k}^{*}$, we only prove the convergence for the biomass fractions $M_{1}^{*}, \ldots, M_{l}^{*}$. The functions $M_{j}^{\epsilon}$ are weak solutions of the auxiliary systems (7). Consequently, the equalities

$$
\begin{aligned}
\int_{\Omega} M_{j}^{\epsilon}(x, T) \varphi(x) d x & -\int_{\Omega} M_{j, 0}(x) \varphi(x) d x+d \int_{Q_{T}} D_{\epsilon}\left(M^{\epsilon}(x, t)\right) \nabla M_{j}^{\epsilon}(x, t) \cdot \nabla \varphi(x) d t d x \\
& =\int_{Q_{T}} f_{j}\left(\underline{S}^{\epsilon}(x, t), \underline{M}^{\epsilon}(x, t)\right) \varphi(x) d t d x, \quad j=1, \ldots, l,
\end{aligned}
$$

are satisfied for all test-functions $\varphi \in C_{0}^{\infty}(\Omega)$ and almost every $T>0$. The family of approximate solutions is uniformly bounded in $L^{\infty}\left(Q_{T}\right)$ by Lemma 1 , and we can immediately pass to the limit 
in all integrals, except for the diffusion term. Hence, it remains to show the convergence of the terms

$$
\int_{Q_{T}} D_{\epsilon}\left(M^{\epsilon}(x, t)\right) \nabla M_{j}^{\epsilon}(x, t) \cdot \nabla \varphi(x) d t d x \rightarrow \int_{Q_{T}} D\left(M^{*}(x, t)\right) \nabla M_{j}^{*}(x, t) \cdot \nabla \varphi(x) d t d x,
$$

$j=1, \ldots, l$, when the regularization parameter $\epsilon$ tends to zero. We remark that the integrals are well-defined by Lemma 3 . We split the difference and treat the domains $Q_{\delta, T}$ and $Q_{\delta, T}^{c}$ separately. To this end we define the integrals

$$
\begin{aligned}
R_{j}^{\epsilon} & :=I_{j}^{\epsilon}+J_{j}^{\epsilon} \\
& :=\int_{Q_{\delta, T}}\left(D_{\epsilon}\left(M^{\epsilon}(x, t)\right) \nabla M_{j}^{\epsilon}(x, t)-D\left(M^{*}(x, t)\right) \nabla M_{j}^{*}(x, t)\right) \cdot \nabla \varphi(x) d t d x \\
& +\int_{Q_{\delta, T}^{c}}\left(D_{\epsilon}\left(M^{\epsilon}(x, t)\right) \nabla M_{j}^{\epsilon}(x, t)-D\left(M^{*}(x, t)\right) \nabla M_{j}^{*}(x, t)\right) \cdot \nabla \varphi(x) d t d x,
\end{aligned}
$$

$j=1, \ldots, l$, and show that the terms $R_{1}^{\epsilon}, \ldots, R_{l}^{\epsilon}$ vanish when $\epsilon$ tends to zero. To estimate the integral $J_{j}^{\epsilon}$ we express the difference as follows

$$
D_{\epsilon}\left(M^{\epsilon}(x, t)\right) \nabla M_{j}^{\epsilon}-D\left(M^{*}\right) \nabla M_{j}^{*}=\left(D_{\epsilon}\left(M^{\epsilon}\right)-D\left(M^{*}\right)\right) \nabla M_{j}^{\epsilon}+D\left(M^{*}\right)\left(\nabla M_{j}^{\epsilon}-\nabla M_{j}^{*}\right) .
$$

For the first term in the integral we obtain

$$
\begin{aligned}
& \left|\left\langle\left(D_{\epsilon}\left(M^{\epsilon}\right)-D\left(M^{*}\right)\right) \nabla M_{j}^{\epsilon}, \nabla \varphi\right\rangle_{L^{2}\left(Q_{\delta, T}^{c} ; \mathbb{R}^{n}\right)}\right| \\
& \leq\left\|D_{\epsilon}\left(M^{\epsilon}\right)-D\left(M^{*}\right)\right\|_{L^{\infty}\left(Q_{\delta, T}^{c}\right)}\left|\left\langle\nabla M_{j}^{\epsilon}, \nabla \varphi\right\rangle_{L^{2}\left(Q_{\delta, T}^{c} ; \mathbb{R}^{n}\right)}\right| \\
& \leq\left\|D_{\epsilon}\left(M^{\epsilon}\right)-D\left(M^{*}\right)\right\|_{L^{\infty}\left(Q_{\delta, T}^{c}\right)}\|\nabla \varphi\|_{L^{2}\left(Q_{\delta, T}^{c} ; \mathbb{R}^{n}\right)}\left\|\nabla M_{j}^{\epsilon}\right\|_{L^{2}\left(Q_{\delta, T}^{c} ; \mathbb{R}^{n}\right)} \\
& \leq C\left\|D_{\epsilon}\left(M^{\epsilon}\right)-D\left(M^{*}\right)\right\|_{L^{\infty}\left(Q_{\delta, T}^{c}\right)}
\end{aligned}
$$

for some constant $C \geq 0$ and $j=1, \ldots, l$. Here, we used the Cauchy-Schwarz inequality and the uniform boundedness of the family of approximate solutions $M_{j}^{\epsilon}$, when restricted to the domain $Q_{\delta, T}^{c}$ in the norm induced by $L^{2}\left((0, T) ; H_{0}^{1}(\Omega)\right)$ (see Lemma 2). The family of solutions $M^{\epsilon}$ of the non-degenerate approximations for the total biomass is uniformly bounded in the Hölder space $C^{\tilde{\alpha}, \frac{\tilde{\alpha}}{2}}\left(Q_{T}\right)$ for some $\tilde{\alpha}>0$ (see [5]), which implies the strong convergence in the space $C\left(Q_{T}\right)$. Furthermore, the solutions of the auxiliary systems satisfy the uniform estimate $M^{\epsilon} \leq 1-\eta$ in $Q_{T}$, and we conclude that $M^{*} \leq 1-\eta$ in $Q_{T}$. On the interval $[0,1-\eta]$ the truncated function $D_{\epsilon}:[0,1-\eta] \rightarrow \mathbb{R}$ converges uniformly to the function $D$ when $\epsilon$ tends to zero. Therefore, splitting the remaining term

$$
\left\|D_{\epsilon}\left(M^{\epsilon}\right)-D\left(M^{*}\right)\right\|_{L^{\infty}\left(Q_{\delta, T}^{c}\right)} \leq\left\|D_{\epsilon}\left(M^{\epsilon}\right)-D_{\epsilon}\left(M^{*}\right)\right\|_{L^{\infty}\left(Q_{\delta, T}^{c}\right)}+\left\|D_{\epsilon}\left(M^{*}\right)-D\left(M^{*}\right)\right\|_{L^{\infty}\left(Q_{\delta, T}^{c}\right)}
$$

we see that it vanishes when $\epsilon$ tends to zero.

Finally, the convergence of the second integral in $J_{j}^{\epsilon}$,

$$
\left\langle D\left(M^{*}\right) \nabla \varphi, \nabla M_{j}^{\epsilon}-\nabla M_{j}^{*}\right\rangle_{L^{2}\left(Q_{\delta, T}^{c} ; \mathbb{R}^{n}\right)}
$$

is an immediate consequence of Lemma 2. Indeed, restricted to the domain $Q_{\delta, T}^{c}$ the family of approximate solutions is uniformly bounded in the norm induced by $L^{2}\left((0, T) ; H_{0}^{1}(\Omega)\right)$, which 
implies the weak convergence in this space. Since the diffusion coefficient $D\left(M^{*}\right)$ belongs to $L^{\infty}\left(Q_{T}\right)$ by Lemma 1 the product $D\left(M^{*}\right) \nabla \varphi$ defines an element in the dual space and implies the convergence of the integral. Summarizing the above estimates we conclude that for every $\mu>0$ there exists $\epsilon_{0}>0$, which is independent of $\delta$, such that the terms $\left|J_{j}^{\epsilon}\right|<\mu$ for all $\epsilon<\epsilon_{0}$ and $j=1, \ldots, l$.

It remains to estimate the integrals $I_{1}^{\epsilon}, \ldots, I_{k}^{\epsilon}$. We recall that for sufficiently small $\epsilon_{0}>0$ the domain $Q_{\delta, T}$ was defined as the subset of $Q_{T}$ where the approximate solutions for the total biomass density satisfy $M^{\epsilon}<\delta$ for all $\epsilon<\epsilon_{0}$. For $\epsilon<\min \left\{\epsilon_{0}, \delta\right\}$ we therefore obtain

$$
D_{\epsilon}\left(M^{\epsilon}(x, t)\right)=\frac{\left(M^{\epsilon}(x, t)+\epsilon\right)^{a}}{\left(1-M^{\epsilon}(x, t)\right)^{b}} \leq \frac{(2 \delta)^{a}}{(1-\delta)^{b}} \quad \forall(x, t) \in Q_{\delta, T} .
$$

Furthermore, the products $\sqrt{D_{\epsilon}\left(M^{\epsilon}\right)} \nabla M_{j}^{\epsilon}, j=1, \ldots, l$, are uniformly bounded in $L^{2}\left(Q_{T} ; \mathbb{R}^{n}\right)$ by Lemma 3, which allows us to use Hölder's inequality to estimate the integral

$$
\begin{aligned}
& \left|\int_{Q_{\delta, T}} D_{\epsilon}\left(M^{\epsilon}(x, t)\right) \nabla M_{j}^{\epsilon}(x, t) \cdot \nabla \varphi(x) d t d x\right| \\
& \leq\left\|\sqrt{D_{\epsilon}\left(M^{\epsilon}(x, t)\right)} \nabla M_{j}^{\epsilon}\right\|_{L^{2}\left(Q_{T} ; \mathbb{R}^{n}\right)}\left\|\sqrt{D_{\epsilon}\left(M^{\epsilon}\right)} \nabla \varphi\right\|_{L^{2}\left(Q_{\delta, T} ; \mathbb{R}^{n}\right)} \\
& \leq C\left(\int_{Q_{\delta, T}} D_{\epsilon}\left(M^{\epsilon}(x, t)\right)\|\nabla \varphi(x)\|^{2} d t d x\right)^{\frac{1}{2}} \leq C \frac{(3 \delta)^{\frac{a}{2}}}{(1-2 \delta)^{\frac{b}{2}}}\|\varphi\|_{L^{2}\left((0, T) ; H^{1}(\Omega)\right)}^{2},
\end{aligned}
$$

for some constant $C \geq 0$ and $j=1, \ldots, l$. Estimating the second term of the integral $I_{j}^{\epsilon}$ in the same way we obtain

$$
\begin{aligned}
\left|I_{\epsilon}\right| \leq & \int_{Q_{\delta, T}}\left|D_{\epsilon}\left(M^{\epsilon}(x, t)\right) \nabla M_{j}^{\epsilon}(x, t) \cdot \nabla \varphi(x)\right| d t d x \\
& +\int_{Q_{\delta, T}}\left|D\left(M^{*}(x, t)\right) \nabla M_{j}^{*}(x, t) \cdot \nabla \varphi(x)\right| d t d x \leq C \frac{(2 \delta)^{\frac{a}{2}}}{(1-\delta)^{\frac{b}{2}}},
\end{aligned}
$$

for some constant $C \geq 0$ and $j=1, \ldots, l$.

To conclude the proof of the theorem let $\mu>0$ be arbitrary. We first choose $\delta>0$ and a corresponding $\epsilon_{1}>0$ such that

$$
\left|I_{j}^{\epsilon}\right|<\frac{\mu}{2 l} \quad \forall \epsilon<\epsilon_{1}, j=1, \ldots, l .
$$

According to the first part of the proof there exists $\epsilon_{0}>0$, which does not dependent on $\delta>0$, such that the integrals

$$
\left|J_{j}^{\epsilon}\right|<\frac{\mu}{2 l} \quad \forall \epsilon<\epsilon_{0}, j=1, \ldots, l .
$$

Consequently, we obtain

$$
\sum_{j=1}^{l}\left|R_{\epsilon}\right| \leq \sum_{j=1}^{l}\left|I_{\epsilon}\right|+\sum_{j=1}^{l}\left|J_{\epsilon}\right|<\mu \quad \forall \epsilon<\min \left\{\epsilon_{0}, \epsilon_{1}\right\} .
$$

This proves that the limit $\left(\underline{S}^{*}, \underline{M}^{*}\right)$ is a solution of System (5) in the sense of Definition 1 . 
4.2. Uniqueness. In this paragraph we prove the uniqueness and $L^{2}(\Omega)$-Lipschitz-continuity of solutions with respect to initial data.

We recall that if the initial data belongs to class $(6)$, then there exists a constant $\eta \in(0,1)$ such that the total biomass satisfies

$$
0 \leq M(x, t) \leq 1-\eta, \quad(x, t) \in \Omega \times \mathbb{R}_{+} .
$$

Consequently, we obtain

$$
0 \leq D(M(x, t))=\frac{(M(x, t))^{a}}{(1-M(x, t))^{b}} \leq \frac{1}{(1-M(x, t))^{b}} \leq \frac{1}{\eta^{b}}, \quad(x, t) \in \Omega \times \mathbb{R}_{+},
$$

which shows that the diffusion coefficient $D(M)$ for the biomass components is non-negative and satisfies $D(M) \in L^{\infty}\left(\Omega \times \mathbb{R}_{+}\right)$.

Theorem 6. Let the initial data belong to the class (6) and Hypotheses $\left(\mathrm{A}_{1}\right)$ and $\left(\mathrm{A}_{2}\right)$ be satisfied. Then, there exists a unique non-negative weak solution $(\underline{S}, \underline{M})$ of the multi-component biofilm model (5).

Proof. The existence of solutions follows from Theorem 5 . We assume that $(\underline{S}, \underline{M})$ and $(\underline{\widehat{S}}, \underline{\widehat{M}})$ are two such solutions of System (5) corresponding to initial data $\left(\underline{S}_{0}, \underline{M}_{0}\right)$ and define the differences $u_{i}:=S_{i}-\widehat{S}_{i}$ and $v_{j}:=M_{j}-\widehat{M}_{j}$, where $i=1, \ldots, k, j=1, \ldots, l$. Then, $u_{i}$ belongs to the space $L^{2}\left((0, T) ; H_{0}^{1}(\Omega)\right), v_{j}$ satisfies $D(M(\cdot, t)) \nabla v_{j}(\cdot, t) \in L^{2}\left(\Omega ; \mathbb{R}^{n}\right)$ for almost every $t \in(0, T]$ and $\partial_{t} u_{i}, \partial_{t} v_{j} \in L^{2}\left((0, T) ; H^{-1}(\Omega)\right)$ for every $T>0$. Moreover, since by $\left(A_{2}\right)$ there exists a unique solution for the total biomass $M$ the functions $u_{i}$ and $v_{j}$ satisfy the system

$$
\begin{aligned}
& \partial_{t} u_{i}=d_{i} \Delta u_{i}+f_{i}(\underline{S}, \underline{M})-f_{i}(\underline{\widehat{S}}, \underline{\widehat{M}}) \quad \Omega \times(0, \infty), \quad i=1, \ldots, k, \\
& \partial_{t} v_{j}=d \nabla \cdot\left(D(M) \nabla v_{j}\right)+g_{j}(\underline{S}, \underline{M})-g_{j}(\underline{\widehat{S}}, \underline{\widehat{M}}) \quad \Omega \times(0, \infty), \quad j=1, \ldots, l,
\end{aligned}
$$

with initial and boundary conditions

$$
\begin{array}{rr}
\left.u_{i}\right|_{\partial \Omega}=\left.v_{j}\right|_{\partial \Omega}=0 & \partial \Omega \times[0, \infty), \\
\left.u_{i}\right|_{t=0}=\left.v_{j}\right|_{t=0}=0 & \Omega \times\{0\},
\end{array}
$$

where $i=1, \ldots, k, j=1, \ldots, l$. If we multiply the first equation by $u_{i}$ and integrate over $\Omega$, we obtain the estimate

$$
\begin{aligned}
\frac{1}{2} \frac{d}{d t}\left\|u_{i}(\cdot, t)\right\|_{L^{2}(\Omega)}^{2}= & -d_{i}\left\|\nabla u_{i}(\cdot, t)\right\|_{L^{2}\left(\Omega ; \mathbb{R}^{n}\right)}^{2} \\
& +\left\langle f_{i}(\underline{S}(\cdot, t), \underline{M}(\cdot, t))-f_{i}(\underline{\widehat{S}}(\cdot, t), \underline{\widehat{M}}(\cdot, t)), u_{i}(\cdot, t)\right\rangle_{L^{2}(\Omega)} \\
\leq & c\left(\sum_{i=1}^{k}\left\|u_{i}(\cdot, t)\right\|_{L^{2}(\Omega)}^{2}+\sum_{j=1}^{l}\left\|v_{j}(\cdot, t)\right\|_{L^{2}(\Omega)}^{2}\right),
\end{aligned}
$$


for some constant $c \geq 0$, where we used Hypothesis $\left(\mathrm{A}_{2}\right)$. Moreover, multiplying the second equation by $v_{j}$ and integrating over $\Omega$ yields

$$
\begin{aligned}
\frac{1}{2} \frac{d}{d t}\left\|v_{j}(\cdot, t)\right\|_{L^{2}(\Omega)}^{2}= & -d\left\langle D(M(x, t)) \nabla v_{j}(\cdot, t), \nabla v_{j}(\cdot, t)\right\rangle_{L^{2}\left(\Omega ; \mathbb{R}^{n}\right)} \\
& +\left\langle g_{j}(\underline{S}(\cdot, t), \underline{M}(\cdot, t))-g_{j}(\underline{\widehat{S}}(\cdot, t), \widehat{\widehat{M}}(\cdot, t)), v_{j}(\cdot, t)\right\rangle_{L^{2}(\Omega)} \\
\leq & c\left(\sum_{i=1}^{k}\left\|u_{i}(\cdot, t)\right\|_{L^{2}(\Omega)}^{2}+\sum_{j=1}^{l}\left\|v_{j}(\cdot, t)\right\|_{L^{2}(\Omega)}^{2}\right)
\end{aligned}
$$

where we used Assumption $\left(\mathrm{A}_{2}\right)$ in the last inequality.

Adding the inequalities for $i=1, \ldots, k$ and $j=1, \ldots, l$ yields

$$
\frac{d}{d t}\left(\sum_{i=1}^{k}\left\|u_{i}(., t)\right\|_{L^{2}(\Omega)}^{2}+\sum_{j=1}^{l}\left\|v_{j}(., t)\right\|_{L^{2}(\Omega)}^{2}\right) \leq C\left(\sum_{i=1}^{k}\left\|u_{i}(., t)\right\|_{L^{2}(\Omega)}^{2}+\sum_{j=1}^{l}\left\|v_{j}(., t)\right\|_{L^{2}(\Omega)}^{2}\right),
$$

for some constant $C \geq 0$. Invoking Gronwall's Lemma and using the initial conditions $\left.u_{i}\right|_{t=0}=$ $\left.v_{j}\right|_{t=0}=0$, we conclude that $\left\|u_{i}(\cdot, t)\right\|_{L^{2}(\Omega)}=\left\|v_{j}(\cdot, t)\right\|_{L^{2}(\Omega)}=0$ for all $t>0$ and $i=1, \ldots, k, j=$ $1, \ldots, l$.

We remark that the proof of Theorem 6 implies the Lipschitz-continuity of the solutions with respect to initial data.

Corollary 1. Let the assumptions of the previous theorem be satisfied. If $(\underline{S}, \underline{M})$ and $(\underline{\widehat{S}}, \underline{\widehat{M}})$ are two solutions of System (5) corresponding to initial data $\left(\underline{S}_{0}, \underline{M}_{0}\right)$ and $\left(\underline{\widehat{S}}_{0}, \widehat{M}_{0}\right)$, then

$$
\begin{aligned}
& \sum_{i=1}^{k}\left\|S_{i}(\cdot, t)-\widehat{S}_{i}(\cdot, t)\right\|_{L^{2}(\Omega)}^{2}+\sum_{j=1}^{l}\left\|M_{j}(\cdot, t)-\widehat{M}_{j}(\cdot, t)\right\|_{L^{2}(\Omega)}^{2} \\
& \leq e^{C t}\left(\sum_{i=1}^{k}\left\|S_{i 0}-\widehat{S}_{i 0}\right\|_{L^{2}(\Omega)}^{2}+\sum_{j=1}^{l}\left\|M_{j 0}-\widehat{M}_{j 0}\right\|_{L^{2}(\Omega)}^{2}\right),
\end{aligned}
$$

for some constant $C \geq 0$.

Proof. The estimate follows immediately from the last inequality in the proof of Theorem 6 and Gronwall's Lemma.

\section{Application to Multicomponent Models}

We show that the general solution theory developed in Section 4 applies to the multicomponent models of Section 3 . To this end it suffices to verify Hypotheses $\left(A_{1}\right)$ and $\left(A_{2}\right)$ in each particular case. Hypothesis $\left(A_{1}\right)$ implies the existence of solutions. If additionally Hypothesis $\left(A_{2}\right)$ is satisfied, then the solutions are unique. 
5.1. Antibiotic Disinfection of Biofilms. The non-degenerate approximations for the antibiotics model are defined as the system (2), where the diffusion coefficient $D(M)$ for the biomass fractions is replaced by the regularized function $D_{\epsilon}(M)$, i.e.,

$$
\begin{aligned}
\partial_{t} S & =d_{S} \Delta S-k_{1} \frac{S X}{k_{2}+S} & & \Omega \times(0, \infty), \\
\partial_{t} B & =d_{B} \Delta B-\zeta_{1} B X & & \Omega \times(0, \infty), \\
\partial_{t} X & =d \nabla \cdot\left(D_{\epsilon}(M) \nabla X\right)+k_{3} \frac{S X}{k_{2}+S}-k_{4} X-\zeta_{2} B X & & \Omega \times(0, \infty), \\
\partial_{t} Y & =d \nabla \cdot\left(D_{\epsilon}(M) \nabla Y\right)+\zeta_{2} B X & & \Omega \times(0, \infty) .
\end{aligned}
$$

To abbreviate notations we introduce the functions

$$
\begin{array}{lrl}
f_{1}(S, B, X, Y):=-k_{1} \frac{S X}{k_{2}+S}, & f_{2}(S, B, X, Y):=-\zeta_{1} B X, \\
g_{1}(S, B, X, Y):=k_{3} \frac{S X}{k_{2}+S}-k_{4} X-\zeta_{2} B X, & g_{2}(S, B, X, Y):=\zeta_{2} B X .
\end{array}
$$

Proposition 2. We assume the functions $S_{r}$ and $B_{r}$ are non-negative and belong to $L^{\infty}(\partial \Omega)$, the initial data $S_{0}, B_{0}, X_{0}, Y_{0}$ belong to the class $L^{\infty}(\Omega)$, are non-negative in $\Omega$ and satisfy

$$
\begin{array}{ll}
S_{0}, B_{0} \in L^{\infty}(\Omega) \cap H^{1}(\Omega), & \left.S_{0}\right|_{\partial \Omega}=S_{r},\left.B_{0}\right|_{\partial \Omega}=B_{r}, \\
X_{0}, Y_{0}, F\left(M_{0}\right) \in H_{0}^{1}(\Omega), & \left\|M_{0}\right\|_{L^{\infty}(\Omega)}<1 .
\end{array}
$$

Then, there exists $\epsilon_{0}>0$ such that the solutions $\left(S^{\epsilon}, B^{\epsilon}, X^{\epsilon}, Y^{\epsilon}\right)$ of the non-degenerate approximations for the antibiotics model are non-negative and uniformly bounded for all $\epsilon<\epsilon_{0}$.

Proof. We observe that the interaction functions satisfy

$$
f_{1}(0, B, X, Y)=f_{2}(S, 0, X, Y)=g_{1}(S, B, 0, Y)=0,
$$

and all components of the solution take non-negative values on the boundary $\partial \Omega$. Moreover, the initial data $S_{0}, B_{0}$ and $X_{0}$ are non-negative in $\Omega$, which implies that the constant zero is a subsolution for $S^{\epsilon}, B^{\epsilon}$ and $X^{\epsilon}$, and the non-negativity of these components follows from a comparison principle for parabolic equations (e.g., see [2]). The non-negativity of $B^{\epsilon}$ and $X^{\epsilon}$ implies that

$$
g_{2}(S, B, X, 0) \geq 0,
$$

and we conclude by the same arguments that the biomass fraction $Y^{\epsilon}$ is non-negative.

To show the uniform boundedness of the substrate concentrations $S^{\epsilon}$ and $B^{\epsilon}$ we define the constants $S_{\max }:=\max \left\{\left\|S_{0}\right\|_{L^{\infty}(\Omega)},\left\|S_{r}\right\|_{L^{\infty}(\partial \Omega)}\right\}$ and $B_{\max }:=\max \left\{\left\|B_{0}\right\|_{L^{\infty}(\Omega)},\left\|B_{r}\right\|_{L^{\infty}(\partial \Omega)}\right\}$. We observe that $\left.S_{\max }\right|_{\partial \Omega} \geq S_{r}=\left.S_{\epsilon}\right|_{\partial \Omega},\left.B_{\max }\right|_{\partial \Omega} \geq B_{r}=\left.B_{\epsilon}\right|_{\partial \Omega},\left.S_{\max }\right|_{t=0} \geq S_{0}=\left.S_{\epsilon}\right|_{t=0}$, $\left.B_{\max }\right|_{t=0} \geq B_{0}=\left.B_{\epsilon}\right|_{t=0}$ and

$$
\begin{aligned}
\partial_{t} S_{\max }-d_{S} \Delta S_{\max }+\kappa_{1} \frac{S_{\max } X^{\epsilon}}{k_{2}+S_{\max }} & =\kappa_{1} \frac{S_{\max } X^{\epsilon}}{k_{2}+S_{\max }} \geq 0, \\
\partial_{t} B_{\max }-d_{B} \Delta B_{\max }+\zeta_{1} B_{\max } X^{\epsilon} & =\zeta_{1} B_{\max } X^{\epsilon} \geq 0,
\end{aligned}
$$


where we used the non-negativity of $X^{\epsilon}$. This shows that $S_{\max }$ and $B_{\max }$ are upper solutions for $S^{\epsilon}$ and $B^{\epsilon}$, and the parabolic comparison principle implies the uniform boundedness of $S^{\epsilon}$ and $B^{\epsilon}$ (see [2]). To conclude the proof it remains to show the uniform boundedness of the biomass fractions $X^{\epsilon}$ and $Y^{\epsilon}$.

We introduce the barrier function $X_{\varphi}:=1+\varphi$, where $\varphi$ is the solution of the elliptic problem

$$
\begin{aligned}
\Delta \varphi(x) & =-1, & x \in \Omega, \\
\left.\varphi\right|_{\partial \Omega}(x) & =0, & x \in \partial \Omega .
\end{aligned}
$$

The maximum principle implies that $\varphi \geq 0$ in $\Omega$ and

$$
1 \leq X_{\varphi}(x) \leq 1+C, \quad x \in \Omega
$$

for some constant $C \geq 0$. Furthermore, we observe that $X_{0}=\left.X^{\epsilon}\right|_{t=0} \leq\left. X_{\varphi}\right|_{t=0}, 0=\left.X^{\epsilon}\right|_{\partial \Omega} \leq$ $\left.X_{\varphi}\right|_{\partial \Omega}$ and

$$
\begin{aligned}
& \partial_{t} X_{\varphi}-d \nabla \cdot\left(D_{\epsilon}\left(X_{\varphi}+Y^{\epsilon}\right) \nabla X_{\varphi}\right)-k_{3} \frac{S^{\epsilon} X_{\varphi}}{k_{2}+S^{\epsilon}}+k_{4} X_{\varphi}+\zeta_{2} B X_{\varphi} \\
= & \frac{d}{\epsilon^{b}}-k_{3} \frac{S^{\epsilon} X_{\varphi}}{k_{2}+S^{\epsilon}}+k_{4} X_{\varphi}+\zeta_{2} B X_{\varphi} \geq \frac{d}{\epsilon^{b}}-k_{3} X_{\varphi}+k_{4} X_{\varphi}+\zeta_{2} B X_{\varphi} \geq \frac{d}{\epsilon^{b}}-k_{3}(1+C) \\
\geq & 0=\partial_{t} X^{\epsilon}-d \nabla \cdot\left(D_{\epsilon}\left(X^{\epsilon}+Y^{\epsilon}\right) \nabla X^{\epsilon}\right)-k_{3} \frac{S^{\epsilon} X^{\epsilon}}{k_{2}+S^{\epsilon}}+k_{4} X^{\epsilon}+\zeta_{2} B^{\epsilon} X^{\epsilon},
\end{aligned}
$$

for all sufficiently small $\epsilon>0$. This shows that $X_{\varphi}$ is an upper solution for the biomass fraction $X^{\epsilon}$. Similarly, we can show that $Y_{\varphi}:=1+\varphi$ is a barrier function for the approximations of the inert biomass $Y^{\epsilon}$. The uniform boundedness of the biomass fractions $X^{\epsilon}$ and $Y^{\epsilon}$ now follows from a parabolic comparison principle (e.g., [2]).

Theorem 7. Let the hypothesis of Proposition 2 be satisfied. Then, there exists a solution of the antibiotics model 22], and it belongs to the class

$$
\begin{aligned}
& S, B, X, Y \in C\left([0, T] ; L^{2}(\Omega)\right) \cap L^{\infty}\left(Q_{T}\right), \\
& S, B \in L^{2}\left((0, T) ; H^{1}(\Omega)\right), \\
& D(M) \nabla X, D(M) \nabla Y \in L^{2}\left((0, T) ; L^{2}\left(\Omega ; \mathbb{R}^{n}\right)\right) .
\end{aligned}
$$

Proof. By Proposition 2 the hypotheses $\left(A_{1}\right)$ is satisfied, and the statement is an immediate consequence of Theorem.

The reaction functions satisfy the hypothesis in $\left(A_{2}\right)$, but we are currently not able to prove the uniqueness of the total biomass $M=X+Y$ for the antibiotics model and the uniqueness of solutions remains open. 
However, for the following modified model (we do not focus here on its biological relevance) a uniqueness result can be established:

$$
\begin{aligned}
& \partial_{t} S=d_{S} \Delta S-k_{1} \frac{S(X+Y)}{k_{2}+S}=d_{S} \Delta S+\tilde{f}_{1}(S, B, X, Y), \\
& \partial_{t} B=d_{B} \Delta B-\zeta_{1} B X=d_{B} \Delta B+f_{2}(S, B, X, Y), \\
& \partial_{t} X=d \nabla \cdot\left(D_{\epsilon}(M) \nabla X\right)+k_{3} \frac{S(X+Y)}{k_{2}+S}-k_{4} X-\zeta_{2} B X=d \nabla \cdot\left(D_{\epsilon}(M) \nabla X\right)+\tilde{g}_{1}(S, B, X, Y), \\
& \partial_{t} Y=d \nabla \cdot\left(D_{\epsilon}(M) \nabla Y\right)+\zeta_{2} B X+k_{4} X=d \nabla \cdot\left(D_{\epsilon}(M) \nabla Y\right)+\tilde{g}_{2}(S, B, X, Y) .
\end{aligned}
$$

Proposition 3. The total biomass of the modified model is unique and the functions $\tilde{f}_{1}, f_{2}, \tilde{g}_{1}$ and $\tilde{g}_{2}$ satisfy the hypotheses in $\left(A_{2}\right)$.

Proof. Adding the governing equations for the biomass fractions $X$ and $Y$ we obtain the prototype biofilm growth model for the total biomass $M$ and nutrient $S$ in Section 2, It was shown in [12] that this system possesses a unique solution. Moreover, let $D$ be a bounded subset of $\mathbb{R}_{+}^{4}$ and $\left(S_{1}, B_{1}, X_{1}, Y_{1}\right),\left(S_{2}, B_{2}, X_{2}, Y_{2}\right) \in D$. We observe that

$$
\begin{aligned}
\tilde{f}_{1}\left(S_{1}, B_{1}, X_{1}, Y_{1}\right) & -\tilde{f}_{1}\left(S_{2}, B_{2}, X_{2}, Y_{2}\right)=-k_{1}\left(\frac{S_{1} X_{1}}{k_{2}+S_{1}}-\frac{S_{2} X_{2}}{k_{2}+S_{2}}\right)-k_{1}\left(\frac{S_{1} Y_{1}}{k_{2}+S_{1}}-\frac{S_{2} Y_{2}}{k_{2}+S_{2}}\right) \\
= & -k_{1}\left(\frac{S_{1}}{k_{2}+S_{1}}\left(X_{1}-X_{2}\right)+X_{2}\left(\frac{S_{1}}{k_{2}+S_{1}}-\frac{S_{2}}{k_{2}+S_{2}}\right)\right) \\
& -k_{1}\left(\frac{S_{1}}{k_{2}+S_{1}}\left(Y_{1}-Y_{2}\right)+Y_{2}\left(\frac{S_{1}}{k_{2}+S_{1}}-\frac{S_{2}}{k_{2}+S_{2}}\right)\right) \\
= & -k_{1}\left(\frac{S_{1}}{k_{2}+S_{1}}\left(X_{1}-X_{2}\right)+\frac{k_{2} X_{2}}{\left(k_{2}+S_{1}\right)\left(k_{2}+S_{2}\right)}\left(S_{1}-S_{2}\right)\right) \\
& -k_{1}\left(\frac{S_{1}}{k_{2}+S_{1}}\left(Y_{1}-Y_{2}\right)+\frac{k_{2} Y_{2}}{\left(k_{2}+S_{1}\right)\left(k_{2}+S_{2}\right)}\left(S_{1}-S_{2}\right)\right) \\
f_{2}\left(S_{1}, B_{1}, X_{1}, Y_{1}\right) & -f_{2}\left(S_{2}, B_{2}, X_{2}, Y_{2}\right)=-\zeta_{1}\left(B_{1} X_{1}-B_{2} X_{2}\right) \\
= & -\zeta_{1} X_{1}\left(B_{1}-B_{2}\right)+B_{2}\left(X_{1}-X_{2}\right),
\end{aligned}
$$

which shows that the functions $\tilde{f}_{1}$ and $f_{2}$ satisfy Hypothesis $\left(\mathrm{A}_{2}\right)$. Using these representations we can analogously verify the assumption for the functions $\tilde{g}_{1}$ and $\tilde{g}_{2}$.

Theorem 8. Let the hypothesis of Proposition 2 be satisfied. Then, there exists a unique solution of the modified antibiotic model, and it belongs to the class

$$
\begin{aligned}
& S, B, X, Y \in C\left([0, T] ; L^{2}(\Omega)\right) \cap L^{\infty}\left(Q_{T}\right) \\
& S, B \in L^{2}\left((0, T) ; H^{1}(\Omega)\right) \\
& D(M) \nabla X, D(M) \nabla Y \in L^{2}\left((0, T) ; L^{2}\left(\Omega ; \mathbb{R}^{n}\right)\right) .
\end{aligned}
$$

Proof. Hypothesis $\left(A_{1}\right)$ for the modified model can be shown following the arguments in the proof of Proposition 2. By Proposition 3 the hypothesis $\left(A_{2}\right)$ is satisfied, and the statement is an immediate consequence of Theorem 5 and Theorem 6 . 
5.2. Amensalistic Biofilm Control System. The non-degenerate approximations for the probiotics model are obtained by replacing the diffusion coefficient $D(M)$ in the equations for the biomass fractions in (3) by the regularized function $D_{\epsilon}(M)$, i.e.,

$$
\begin{aligned}
& \partial_{t} C=d_{C} \Delta C+\alpha_{1} X\left(\zeta_{1}-C\right)+\alpha_{2} Y\left(\zeta_{1}-C\right) \quad \Omega \times(0, \infty), \\
& \partial_{t} P=d_{P} \Delta P+\alpha_{3} C\left(\zeta_{2}-P\right) \quad \Omega \times(0, \infty), \\
& \partial_{t} X=d \nabla \cdot\left(D_{\epsilon}(M) \nabla X\right)+\mu_{1} \psi_{1}(C, P) X \quad \Omega \times(0, \infty), \\
& \partial_{t} Y=d \nabla \cdot\left(D_{\epsilon}(M) \nabla Y\right)+\mu_{2} \psi_{2}(C, P) Y \quad \Omega \times(0, \infty), \\
& \partial_{t} Z=d \nabla \cdot\left(D_{\epsilon}(M) \nabla Z\right)-\min \left\{0, \mu_{1} \psi_{1}(C, P) X\right\}-\min \left\{0, \mu_{2} \psi_{2}(C, P) Y\right\} \quad \Omega \times(0, \infty) .
\end{aligned}
$$

To abbreviate notations we define the functions

$$
\begin{array}{lr}
f_{1}(C, P, X, Y, Z):=\alpha_{1} X\left(\zeta_{1}-C\right)+\alpha_{2} Y\left(\zeta_{1}-C\right), & f_{2}(C, P, X, Y, Z):=\alpha_{3} C\left(\zeta_{2}-P\right), \\
g_{1}(C, P, X, Y, Z):=\mu_{1} \psi_{1}(C, P) X, & g_{2}(C, P, X, Y, Z):=\mu_{2} \psi_{2}(C, P) Y, \\
g_{3}(C, P, X, Y, Z):=-\min \left\{0, \mu_{1} \psi_{1}(C, P) X\right\}-\min \left\{0, \mu_{2} \psi_{2}(C, P) Y\right\} .
\end{array}
$$

Proposition 4. Let the functions $C_{r}$ and $P_{r}$ be non-negative, belong to $L^{\infty}(\partial \Omega)$ and satisfy

$$
0 \leq C_{r} \leq \zeta_{1}, \quad 0 \leq P_{r} \leq \zeta_{2} .
$$

Moreover, we assume the initial data $C_{0}, P_{0}, X_{0}, Y_{0}, Z_{0}$ belong to the class $L^{\infty}(\Omega)$, are non-negative in $\Omega$ and satisfy

$$
\begin{array}{ll}
C_{0}, P_{0} \in L^{\infty}(\Omega) \cap H^{1}(\Omega), & 0 \leq C_{0} \leq \zeta_{1}, 0 \leq P_{0} \leq \zeta_{2},\left.\quad C_{0}\right|_{\partial \Omega}=C_{r},\left.P_{0}\right|_{\partial \Omega}=P_{r}, \\
X_{0}, Y_{0}, Z_{0}, F\left(M_{0}\right) \in H_{0}^{1}(\Omega), & \left\|M_{0}\right\|_{L^{\infty}(\Omega)}<1 .
\end{array}
$$

Then, there exists $\epsilon_{0}>0$ such that the solutions $\left(C^{\epsilon}, P^{\epsilon}, X^{\epsilon}, Y^{\epsilon}, Z^{\epsilon}\right)$ of the non-degenerate approximations for the probiotics model are non-negative and uniformly bounded for all $\epsilon<\epsilon_{0}$.

Proof. We observe that the interaction functions in the equations for the biomass components satisfy

$$
g_{1}(C, P, 0, Y, Z)=g_{2}(C, P, X, 0, Z)=0, \quad g_{3}(C, P, X, Y, 0) \geq 0,
$$

and $X^{\epsilon}, Y^{\epsilon}$ and $Z^{\epsilon}$ take non-negative values on the boundary $\partial \Omega$. Moreover, the initial data $X_{0}, Y_{0}$ and $Z_{0}$ are non-negative in $\Omega$, which implies that the constant zero is a subsolution for $X^{\epsilon}, Y^{\epsilon}$ and $Z^{\epsilon}$, and the non-negativity of the biomass fractions follows from a comparison principle for parabolic equations. Using the non-negativity of the biomass fractions we observe that

$$
f_{1}(0, P, X, Y, Z) \geq 0, \quad f_{2}(C, 0, X, Y, Z) \geq 0,
$$

which implies that the constant zero is also a subsolution for the substrate concentrations $C^{\epsilon}$ and $P^{\epsilon}$.

The initial and boundary data satisfy $C_{r} \leq \zeta_{1}, P_{r} \leq \zeta_{2}$ and $0 \leq C_{0} \leq \zeta_{1}, 0 \leq P_{0} \leq \zeta_{2}$. Moreover, since

$$
f_{1}\left(\zeta_{1}, P, X, Y, Z\right)=0, \quad f_{2}\left(C, \zeta_{2}, X, Y, Z\right)=0,
$$


the constants $\zeta_{1}$ and $\zeta_{2}$ are upper solutions for the substrate concentrations $C^{\epsilon}$ and $P^{\epsilon}$. To show the uniform boundedness of the biomass fractions we consider the barrier function $X_{\varphi}=1+\varphi$ as in the proof of Proposition 2. It satisfies $X_{0}=\left.X^{\epsilon}\right|_{t=0} \leq\left. X_{\varphi}\right|_{t=0}, 0=\left.X^{\epsilon}\right|_{\partial \Omega} \leq\left. X_{\varphi}\right|_{\partial \Omega}$ and

$$
\begin{aligned}
& \partial_{t} X_{\varphi}-d \nabla \cdot\left(D_{\epsilon}\left(X_{\varphi}+Y^{\epsilon}+Z^{\epsilon}\right) \nabla X_{\varphi}\right)-\mu_{1} \psi_{1}\left(C^{\epsilon}, P^{\epsilon}\right) X_{\varphi} \\
= & \frac{d}{\epsilon^{b}}-\mu_{1} \psi_{1}\left(C^{\epsilon}, P^{\epsilon}\right) X_{\varphi} \geq \frac{d}{\epsilon^{b}}-\mu_{1}(1+\varphi) \geq 0,
\end{aligned}
$$

for all sufficiently small $\epsilon>0$. This shows that $X_{\varphi}$ is an upper solution for the biomass fraction $X^{\epsilon}$. Similarly, we can show that $Y_{\varphi}=1+\varphi$ and $Z_{\varphi}=1+\varphi$ are upper solutions for the biomass fractions $Y^{\epsilon}$ and $Z^{\epsilon}$, which concludes the proof.

Theorem 9. Let the assumptions of Proposition 4 be satisfied. Then, there exists a solution of the probiotics model (3), and it belongs to the class

$$
\begin{aligned}
& C, P, X, Y, Z \in C\left([0, T] ; L^{2}(\Omega)\right) \cap L^{\infty}\left(Q_{T}\right), \\
& C, P \in L^{2}\left((0, T) ; H^{1}(\Omega)\right), \\
& D(M) \nabla X, D(M) \nabla Y, D(M) \nabla Z \in L^{2}\left((0, T) ; L^{2}\left(\Omega ; \mathbb{R}^{n}\right)\right) .
\end{aligned}
$$

Proof. By Proposition 4 the Hypotheses $\left(A_{1}\right)$ is satisfied, and the statement is an immediate consequence Theorem 5 .

Similarly to the antibiotics model, at the moment we cannot prove the uniqueness of the total biomass $M$ for the probiotics model, and the uniqueness of solutions remains open.

5.3. Quorum-Sensing in Patchy Biofilm Communities. We define the non-degenerate approximations for the quorum-sensing model (4) as the system

$$
\begin{array}{rlrl}
\partial_{t} S & =d_{S} \Delta S-k_{1} \frac{S M}{k_{2}+S} & & \Omega \times(0, \infty), \\
\partial_{t} A & =d_{A} \Delta A-\gamma A+\alpha X+(\alpha+\beta) Y & & \Omega \times(0, \infty), \\
\partial_{t} X=d \nabla \cdot\left(D_{\epsilon}(M) \nabla X\right)+k_{3} \frac{X S}{k_{2}+S}-k_{4} X-k_{5}|A|^{m} X+k_{5}|Y| & & \Omega \times(0, \infty), \\
\partial_{t} Y & =d \nabla \cdot\left(D_{\epsilon}(M) \nabla Y\right)+k_{3} \frac{Y S}{k_{2}+S}-k_{4} Y+k_{5}|A|^{m} X-k_{5}|Y| & & \Omega \times(0, \infty),
\end{array}
$$

where the diffusion coefficient $D(M)$ for the biomass fractions is replaced by the regularized function $D_{\epsilon}(M)$. Moreover, to abbreviate notations we introduce the functions

$$
\begin{aligned}
& f_{1}(S, A, X, Y):=-k_{1} \frac{S M}{k_{2}+S}, \quad f_{2}(S, A, X, Y):=-\gamma A+\alpha X+(\alpha+\beta) Y, \\
& g_{1}(S, A, X, Y):=k_{3} \frac{X S}{k_{2}+S}-k_{4} X-k_{5}|A|^{m} X+k_{5}|Y|, \\
& g_{2}(S, A, X, Y):=k_{3} \frac{Y S}{k_{2}+S}-k_{4} Y+k_{5}|A|^{m} X-k_{5}|Y| .
\end{aligned}
$$


Proposition 5. We assume the initial data $S_{0}, A_{0}, X_{0}, Y_{0}$ belong to the class $L^{\infty}(\Omega)$, are nonnegative in $\Omega$ and satisfy

$$
\begin{array}{ll}
S_{0} \in L^{\infty}(\Omega) \cap H^{1}(\Omega), & \left.S_{0}\right|_{\partial \Omega}=1, \\
A_{0}, X_{0}, Y_{0}, F\left(M_{0}\right) \in H_{0}^{1}(\Omega), & \left\|M_{0}\right\|_{L^{\infty}(\Omega)}<1 .
\end{array}
$$

Then, there exists $\epsilon_{0}>0$ such that the solutions $\left(S^{\epsilon}, A^{\epsilon}, X^{\epsilon}, Y^{\epsilon}\right)$ of the non-degenerate approximations for the quorum-sensing model (4) are non-negative and uniformly bounded for all $\epsilon<\epsilon_{0}$.

Proof. The interaction functions in the governing equations for $S^{\epsilon}$ and $X^{\epsilon}$ satisfy

$$
f_{1}(0, A, X, Y)=0, \quad g_{1}(S, A, 0, Y) \geq 0,
$$

all components of the solution take non-negative values on the boundary $\partial \Omega$, and the initial data $S_{0}$ and $X_{0}$ are non-negative in $\Omega$. This implies that the constant zero is a subsolution for $S^{\epsilon}$ and $X^{\epsilon}$, and the non-negativity of these components follows from a comparison principle for parabolic equations (see [2]). The non-negativity of $S^{\epsilon}$ and $X^{\epsilon}$ imply that

$$
g_{2}(S, 0, X, Y) \geq 0, \quad f_{2}(S, A, X, 0) \geq 0
$$

and we conclude by the same arguments that the autoinducer concentration $A^{\epsilon}$ and the biomass fraction $Y^{\epsilon}$ are non-negative.

The particularity of the quorum-sensing model is, that adding the equations for the biomass fractions $X^{\epsilon}$ and $Y^{\epsilon}$ leads to the system

$$
\begin{array}{rlrl}
\partial_{t} S & =d_{S} \Delta S-k_{1} \frac{S M}{k_{2}+S} & \Omega \times(0, \infty), \\
\partial_{t} M & =d \nabla \cdot\left(D_{\epsilon}(M) \nabla M\right)+k_{3} \frac{S M}{k_{2}+S}-k_{4} M & \Omega \times(0, \infty), \\
\left.M\right|_{\partial \Omega} & =0,\left.\quad S\right|_{\partial \Omega}=1 & & \partial \Omega \times[0, \infty), \\
\left.M\right|_{t=0} & =X_{0}+Y_{0},\left.\quad S\right|_{t=0}=S_{0} & & \Omega \times\{0\},
\end{array}
$$

which are the non-degenerate approximations for the prototype biofilm growth model discussed in Section 2. By Proposition 1 and Proposition 6 in [12, the solutions $S^{\epsilon}$ and $M^{\epsilon}$ are uniformly bounded by the constant 1 for all sufficiently small $\epsilon>0$. Since the biomass fractions are nonnegative this implies that $X^{\epsilon}$ and $Y^{\epsilon}$ are uniformly bounded.

Finally, we show that the constant $A_{\max }:=\max \left\{\frac{\alpha+\beta}{\gamma},\left\|A_{0}\right\|_{L^{\infty}(\Omega)}\right\}>1$ is an upper solution for $A^{\epsilon}$. It satisfies $\left.A_{\max }\right|_{\partial \Omega} \geq 0=\left.A^{\epsilon}\right|_{\partial \Omega},\left.A_{\max }\right|_{t=0} \geq A_{0}=\left.A^{\epsilon}\right|_{t=0}$ and

$$
\begin{aligned}
\partial_{t} A_{\max }-d_{A} \Delta A_{\max }+\gamma A_{\max }-\alpha X^{\epsilon}-(\alpha+\beta) Y^{\epsilon} & =\gamma A_{\max }-\alpha X^{\epsilon}-(\alpha+\beta) Y^{\epsilon} \\
& \geq \gamma A_{\max }-\alpha-\beta=0,
\end{aligned}
$$

where we used the assumption $\alpha+\beta>\gamma$ in Section 3.3 .

Proposition 6. There exists a unique solution for the total biomass $M$, and the functions $f_{1}, f_{2}, g_{1}$ and $g_{2}$ satisfy Hypothesis $\left(A_{2}\right)$. 
Adding the equations for the biomass fractions $X$ and $Y$ leads to the prototype biofilm growth model discussed in Section 2, for which the existence and uniqueness of solutions was shown in [12. Moreover, hypothesis $\left(\mathrm{A}_{2}\right)$ is certainly satisfied by the function $f_{2}$, and for the function $f_{1}$ it can be verified as in the proof of Proposition 3. To show the property for the functions $g_{1}$ and $g_{2}$ we observe that

$$
\begin{aligned}
A_{1}^{m} X_{1}-A_{2}^{m} X_{2} & =A_{1}^{m}\left(X_{1}-X_{2}\right)+X_{2}\left(A_{1}^{m}-A_{2}^{m}\right) \\
& =A_{1}^{m}\left(X_{1}-X_{2}\right)+m X_{2}\left(A_{1}-A_{2}\right) \int_{0}^{1}\left(s A_{1}+(1-s) A_{2}\right)^{m-1} d s,
\end{aligned}
$$

which implies that

$$
A_{1}^{m} X_{1}-A_{2}^{m} X_{2} \leq C\left(\left(X_{1}-X_{2}\right)+X_{2}\left(A_{1}-A_{2}\right)\right)
$$

for some constant $C \geq 0$ and $\left(X_{1}, A_{1}\right),\left(X_{2}, A_{2}\right)$ in bounded subsets of $\mathbb{R}_{+}^{2}$. All remaining terms can be decomposed as in the proof of Proposition 3.

Theorem 10. Let the assumptions of Proposition 5 be satisfied. Then, there exists a unique solution of the quorum-sensing model (4), and it belongs to the class

$$
\begin{aligned}
& S, A, X, Y \in C\left([0, T] ; L^{2}(\Omega)\right) \cap L^{\infty}\left(Q_{T}\right), \\
& S, A \in L^{2}\left((0, T) ; H^{1}(\Omega)\right), \\
& D(M) \nabla X, D(M) \nabla Y \in L^{2}\left((0, T) ; L^{2}\left(\Omega ; \mathbb{R}^{n}\right)\right) .
\end{aligned}
$$

Proof. This is an immediate consequence of Proposition 5 , Proposition 6 , Theorem 5 and Theorem 6.

\section{Computer simulations}

For illustration we include computer simulations of the antibiotics model (2), in the form

$$
\begin{array}{ll}
\partial_{t} S=d_{S} \Delta S-k_{1} \frac{S X}{k_{2}+S} & \Omega \times(0, \infty), \\
\partial_{t} B=d_{B} \Delta B-\zeta_{1} B X & \Omega \times(0, \infty), \\
\partial_{t} X=d \nabla \cdot(D(M) \nabla X)+k_{3} \frac{S X}{k_{2}+S}-k_{4} X-\zeta_{2} B X & \Omega \times(0, \infty), \\
\partial_{t} Y=d \nabla \cdot(D(M) \nabla Y)+\zeta_{2} B X & \Omega \times(0, \infty),
\end{array}
$$

with $M=X+Y$ and

$$
D(M)=\frac{M^{a}}{(1-M)^{b}}
$$

and initial and boundary data

$$
\begin{array}{rrrrr}
\left.X\right|_{\partial \Omega}=0, & \left.Y\right|_{\partial \Omega}=0, & \left.S\right|_{\partial \Omega}=S_{r}, & \left.B\right|_{\partial \Omega}=B_{r} & \partial \Omega \times[0, \infty), \\
\left.X\right|_{t=0}=X_{0}, & \left.Y\right|_{t=0}=Y_{0}, & \left.S\right|_{t=0}=S_{0}, & \left.B\right|_{t=0}=B_{0} & \Omega \times\{0\} .
\end{array}
$$

The model parameters are summarized in Table 1. The method of discretization has been described and discussed in some detail in [18, 19]. A standard Finite Volume discretization on a rectangular 
grid is used for treatment of spatial terms, and a semi-implicit time integration method. In every time step this requires the solution of one sparse linear system for each dependent variable. By construction, the system matrices are at least weakly diagonally dominant. The Jacobi preconditioned Conjugate Gradient method [24] is used for their inversion. It is implemented in Fortran and prepared for parallel execution on GPU accelerators using OpenACC, as described in [9].

TABLE 1. Parameters used in the computer simulations of the growth-disinfection model 2 (2)

\begin{tabular}{lccc} 
parameter & symbol & value & units \\
\hline maximum substrate uptake rate & $k_{1}$ & 95238 & $1 / \mathrm{d}$ \\
substrate half saturation concentration & $k_{2}$ & 4 & $\mathrm{gm}^{3}$ \\
maximum growth rate & $k_{3}$ & 6 & $1 / \mathrm{d}$ \\
lysis rate & $k_{4}$ & 0.4 & $1 / \mathrm{d}$ \\
antibiotic uptake rate & $\zeta_{1}$ & 4762 & $1 / \mathrm{d}$ \\
relative disinfection rate & $\zeta_{2}$ & 12 & $\mathrm{~m}^{3} /(\mathrm{gd})$ \\
substrate diffusion coefficient & $d_{S}$ & $10^{-4}$ & $\mathrm{~m}^{2} / \mathrm{d}$ \\
antibiotics diffusion coefficient & $d_{B}$ & $0.15 \cdot 10^{-4}$ & $\mathrm{~m}^{2} / \mathrm{d}$ \\
biomass motility rate & $d$ & $10^{-12}$ & $\mathrm{~m}^{2} / \mathrm{d}$ \\
biofilm exponent & $a$ & 4 & - \\
biofilm exponent & $b$ & 4 & - \\
bulk substrate concentration & $S_{r}$ & 20 & $\mathrm{~g} / \mathrm{m}^{3}$ \\
antibiotics substrate concentration & $B_{r}$ & 1 & $\mathrm{~g} / \mathrm{m}^{3}$ \\
system length & $L$ & $10^{-3}$ & $\mathrm{~m}$ \\
\hline
\end{tabular}

We simulate the case of a biofilm without substratum, i.e. microbial flocs. We place initially five identical spherical flocs in a square domain $\Omega$ of size $L \times L$. One floc is placed in the center of the domain, the other four in the centers of the quadrants. Initially, we solve the biofilm growth model only, i.e. no antibiotics is added, the boundary concentration is kept at 0 . In this case the disinfection model reduces to the single species growth model. At a given disinfection start time $t_{s}$, the boundary antibiotics concentration is switched to 1 and the growth/disinfection model is simulated. We repeat the simulation for different disinfection start times $t_{s}=2.802,4.002,4.402,4.802$. In Figure 2 we plot the following normalized lumped results:

(a) active biomass $X_{\text {total }}(t)=\int_{\Omega} X(t, x) d x$,

(b) inactive biomass $Y_{\text {total }}(t)=\int_{\Omega} Y(t, x) d x$,

(c) amount of antibiotics $A_{\text {total }}(t)=\int_{\Omega} A(t, x) d x$, and

(d) biofilm size $\omega(t)=\int_{X(t)+Y(t)>0} d x$.

We observe that for the smallest disinfection start time $t_{s}=2.802$ the active biomass in the system begins to decline and eventually the biofilm is eradicated. For $t_{s}=4.002$ the active biomass first 


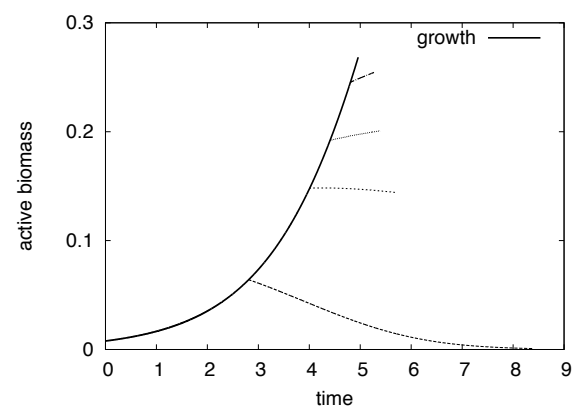

(a)

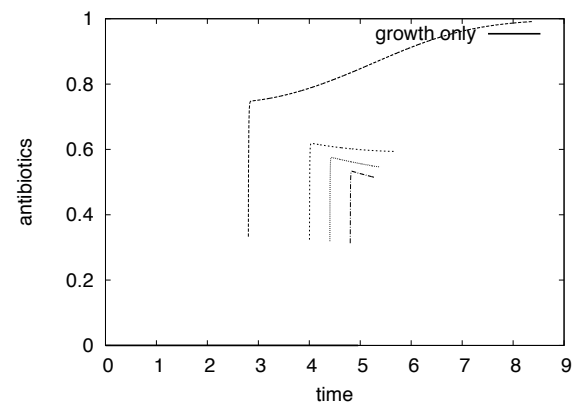

(c)

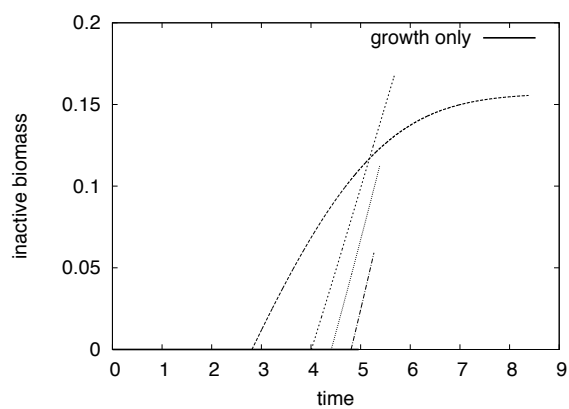

(b)

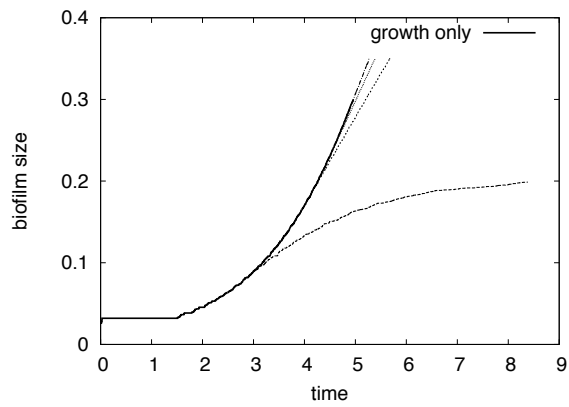

(d)

Figure 2. Simulation of the biofilm disinfection model for different starting times of treatment (lumped results) and for the model without disinfection (growth only, bold line). Shown are (a) active biomass, (b) inactive biomass, (c) antibiotics concentration, (d) biofilm size.

remains at a plateau and then slowly decays. For the remaining larger disinfection start times the active biomass keeps increasing even in the presence of antibiotics. In all cases the amount of inactive biomass increases monotonically. Biofilm size increases in all cases but levels off in the case of the smallest disinfection start time where all active biomass is killed off. The amount of antibiotics in the system increases in the case where treatment is efficient, due to the decline of active biomass that degrades the antibiotics. In all other cases, the antibiotics concentration decreases, indicating that less antibiotics is used up than added.

In Figure 3 we plot for the simulation with disinfection start time $t_{s}=4.402$ for six selected time instances the fraction of active biomass relative to the overall biomass, $Z(t, x)=\frac{X(t, x)}{X(t, x)+Y(t, x)}$, in the biofilm, i.e. in the region $\Omega_{1}(t)=\{x \in \Omega: X(t, x)+Y(t, x)>0\}$. This is a scenario for which the treatment ultimately fails, i.e. the active biomass in the system continues growing. Our results show, that the local efficiency of treatment is not homogeneous. In the outer colonies, closer to substrate and antibiotic source, active biomass declines (note also the slight biomass gradients within these colonies), whereas the colony in the centre remains strongly dominated by active biomass, protected by the outer colonies that degrade the antibiotics. This illustrates the 


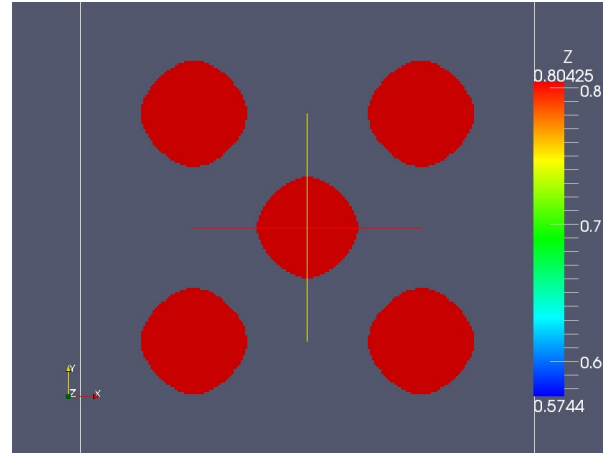

$t=4.404$

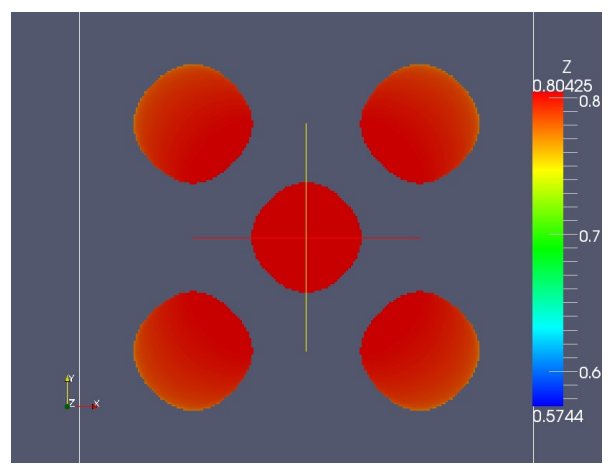

$t=4.804$

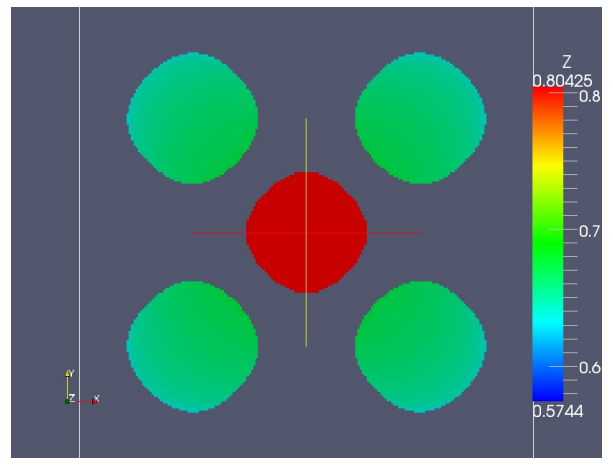

$t=5.204$

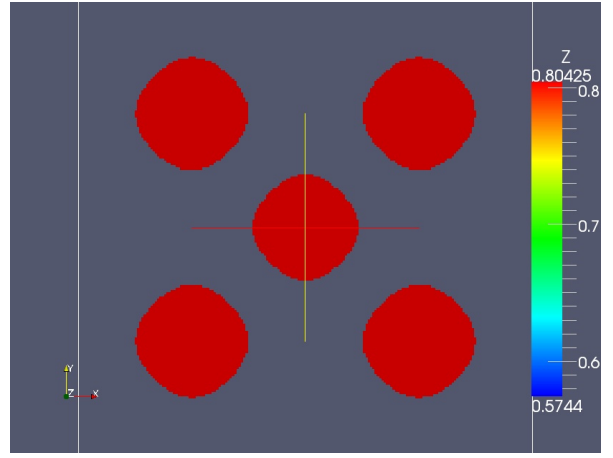

$t=4.604$

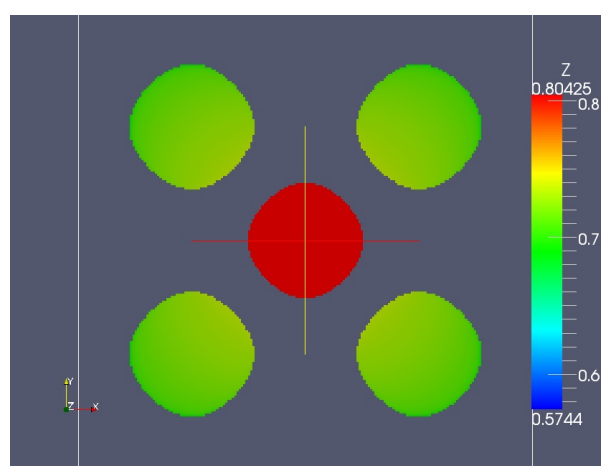

$t=5.004$

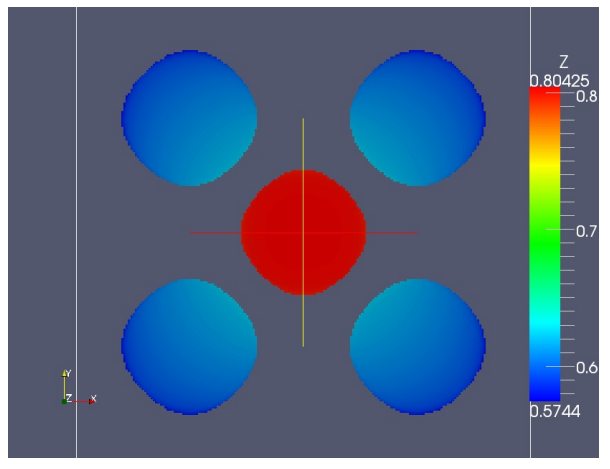

$t=5.388$

FiguRE 3. Simulation of concurrent disinfection and growth: shown is the active biomass at different times $t$. Disinfection was started at $t=4.402$ after a period of growth only. Color coded is the fraction of active biomass $Z=X /(X+Y)$.

role of spatial structure in antibiotic treatment of biofilms. Note that also the outer colonies still have a substantial amount of active biomass at $t=5.388$.

\section{Concluding Remarks}

In this study we provided relatively easy to verify criteria for existence and uniqueness to solutions to a class of multicomponent biofilm models. These generalize and expand earlier results that were previously obtained for specific applications. 
The existence results in the previous section are formulated assuming homogeneous Dirichlet boundary conditions for the biomass components. This situation resembles growing biofilms without substratum, which are commonly called microbial flocs. Such bacterial aggregates enclosed in an EPS matrix are used in wastewater treatment in activated sludge systems and also occur in natural settings [22]. Boundary conditions of mixed type are, however, often more appropriate in applications. Typically, Dirichlet conditions are specified on some part of the boundary, while Neumann or Robin conditions are imposed on the other parts. In particular, the substratum, on which the biofilm grows is impermeable for all dependent variables, which is described by homogeneous Neumann boundary values. The boundary conditions for the dissolved substrates, which describe mechanisms of substrate replenishment and removal are rather uncritical. The well-posedness proof carries over to these more general situations as long as homogeneous Dirichlet conditions are imposed for the biomass fractions on one part of the boundary (for details see Theorem 4.1, [12]). On the other hand, if homogeneous Neumann conditions are assumed for all biomass components and constant Dirichlet conditions for the nutrient concentration, which reflects the situation that no biomass can leave the system and nutrients are constantly added, it was shown that the biomass density reaches the singular value in finite time (Proposition 7 and Proposition 8, [12]).

\section{REFERENCES}

[1] Battin TJ, Sloan WT, Kjelleberg S, Daims H, Head IM, Curtis TP, Eberl L. Microbial landscapes: new paths to biofilm research. Nature Reviews Microbiology. 2007; 5(1): 76-81. DOI:10.1038/nrmicro1556.

[2] Boyadjiev G, Kutev N: Comparison principle for quasilinear elliptic and parabolic systems. Comptes rendus de l'Académie bulgare des Sciences. 2002, 55(1): 9-12.

[3] Chepyzhov VV, Vishik MI. Attractors for Equations of Mathematical Physics. American Mathematical Society: Providence, Rhode Island; 2002.

[4] Costerton JW, Stewart PS, Greenberg EP. Bacterial biofilms: a common cause of persistent infections. Science. 1999; 284: 1318-1322. DOI:10.1126/science.284.5418.1318.

[5] Demaret L, Eberl HJ, Efendiev MA, Lasser R. Analysis and simulation of a meso-scale model of diffusive resistance of bacterial biofilms to penetration of antibiotics. Advances in Mathematical Sciences and Applications. 2008; 18(1): 269-304.

[6] Eberl HJ, Efendiev MA. A transient density-dependent diffusion-reaction model for the limitation of antibiotic penetration in biofilms. Electronic Journal of Differential Equations. 2003; Conference 10; 123-142.

[7] Eberl HJ, Parker DF, van Loosdrecht M. A new deterministic spatio-temporal continuum model for biofilm development. Journal of Theoretical Medicine. 2001; 3(3): 161-175. DOI:10.1080/10273660108833072.

[8] Eberl HJ, Sudarsan R. A brief note on ecological and mechanical views in biofilm modeling. International Journal of Biomathematics and Biostatistics. 2010; 1(1): 33-45.

[9] Eberl HJ, Sudarsan R. OpenACC parallelisation for diffusion problems, applied to temperature distribution on a honeycomb around the bee brood: a worked example using BiCGSTAB. Lecture Notes in Computer Science. 2014; 8385 in 9: 311-321. DOI:10.1007/978-3-642-55195-629.

[10] Efendiev M. Evolution Equations Arising in the Modelling of Life Sciences. International Series of Numerical Mathematics 163. Birkhäuser: Basel; 2013.

[11] Efendiev MA, Demaret L. On the structure of attractors for a class of degenerate reaction-diffusion systems. Advances in Mathematical Sciences and Applications. 2008; 18(2): 105-118. 
[12] Efendiev MA, Eberl HJ, Zelik SV. Existence and longtime behavior of a biofilm model. Communications on Pure and Applied Mathematics. 2009; 8(2): 509-531. DOI:10.3934/cpaa.2009.8.509.

[13] Frederick MR, Kuttler C, Hense BA, Müller J, Eberl HJ. A mathematical model of quorum sensing in patchy biofilm communities with slow background flow. Canadian Applied Math Quarterly. 2010; 18(3): 267-298.

[14] Hense BA, Kuttler C, Müller J, Rothballer M, Hartmann A, Kreft J-U. Does efficiency sensing unify diffusion and quorum sensing?. Nature Reviews Microbiology. 2007; 5(3): 230-239. DOI:10.1038/nrmicro1600.

[15] Khassehkhan H, Efendiev MA, Eberl HJ. A degenerate diffusion-reaction model of an amensalistic biofilm control system: existence and simulation of solutions. Discrete and Continuous Dynamical Systems Series B. 2009; 12(2): 371-388. DOI:10.3934/dcdsb.2009.12.371.

[16] Khassehkhan H, Eberl HJ. Modeling and simulation of a bacterial biofilm that is controlled by $\mathrm{pH}$ and protonated lactic acids. Computational and Mathematical Models in Medicine. 2008; 9(1): 47-67. DOI:10.1080/17486700701797922.

[17] Ladyzenskaja OA, Solonnikov VA, Ural'ceva NN. Linear and Quasi-linear Equations of Parabolic Type. American Mathematical Society: Providence, Rhode Island; 1968.

[18] Muhammad N, Eberl HJ. OpenMP parallelization of a Mickens time-integration scheme for a mixed-culture biofilm model and its performance on multi-core and multi-processor computers. Lecture Notes in Computer Science. 2010; 5976 in 18: 180-195. DOI:10.1007/978-3-642-12659-8-14.

[19] Muhammad N, Eberl HJ. Model parameter uncertainties in a dual-species biofilm competition model affect ecological output parameters much stronger than morphological ones. Mathematical Biosciences. 2011; 233(1): 1-18. DOI:10.1016/j.mbs.2011.05.006.

[20] Müller J, Kuttler C, Hense BA, Rothballer M, Hartmann A. Cell-cell communication by quorum sensing and dimension-reduction. Journal of Mathematical Biology. 2006; 53(4): 672-702. DOI:10.1007/s00285-006-0024-z.

[21] O'Toole GA, Stewart PS. Biofilms strike back. Nature Biotechnology. 2005; 23(11): 1378-1379. DOI:10.1038/nbt1105-1378.

[22] Parsek MR, Greenberg EP. Sociomicrobiology: the connections between quorum sensing and biofilms. Trends in Microbiology. 2005; 13(1): 27-33. DOI:10.1016/j.tim.2004.11.007.

[23] Renardy M., Rogers RC. An Introduction to Partial Differential Equations. Second Edition, Springer-Verlag: New York; 2004.

[24] Saad Y. Iterative Methods for Sparse Linear Systems. Second edition, SIAM: Philadelphia; 2003.

[25] Sonner S. A class of mathematical models describing processes in spatially heterogeneous biofilm communities. Biomath Forum. 2013; 2(2): 1312311, 10 pp.. DOI:10.1002/mma.1475.

[26] Sonner S, Efendiev MA, Eberl HJ. On the well-posedness of a mathematical model of quorum-sensing in patchy biofilm communities. Mathematical Methods in the Applied Sciences. 2011; 34(13): 1667-1684. DOI:10.1002/mma.1475.

[27] Wanner O, Gujer W. A multispecies biofilm model. Biotechnology and Bioengineering. 1986; 28(3): 314-328. DOI:10.1002/bit.260280304. 